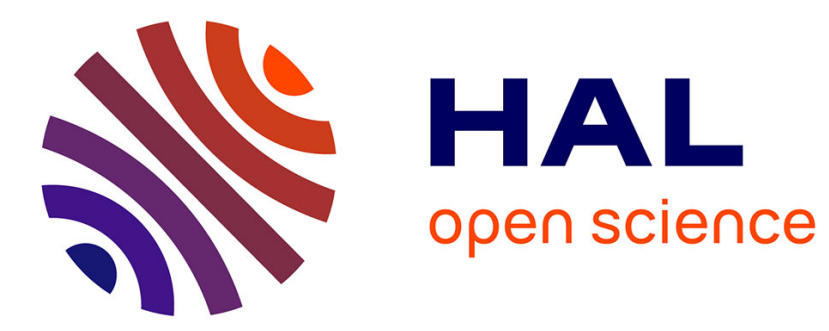

\title{
A mean field theory for diffusion in a dilute multi-component alloy: a new model for the effect of solutes on self-diffusion
}

Maylise Nastar

\section{- To cite this version:}

Maylise Nastar. A mean field theory for diffusion in a dilute multi-component alloy: a new model for the effect of solutes on self-diffusion. Philosophical Magazine, 2005, 85 (32), pp.3767-3794. $10.1080 / 14786430500228390$. hal-00513556

\section{HAL Id: hal-00513556 \\ https://hal.science/hal-00513556}

Submitted on 1 Sep 2010

HAL is a multi-disciplinary open access archive for the deposit and dissemination of scientific research documents, whether they are published or not. The documents may come from teaching and research institutions in France or abroad, or from public or private research centers.
L'archive ouverte pluridisciplinaire HAL, est destinée au dépôt et à la diffusion de documents scientifiques de niveau recherche, publiés ou non, émanant des établissements d'enseignement et de recherche français ou étrangers, des laboratoires publics ou privés. 


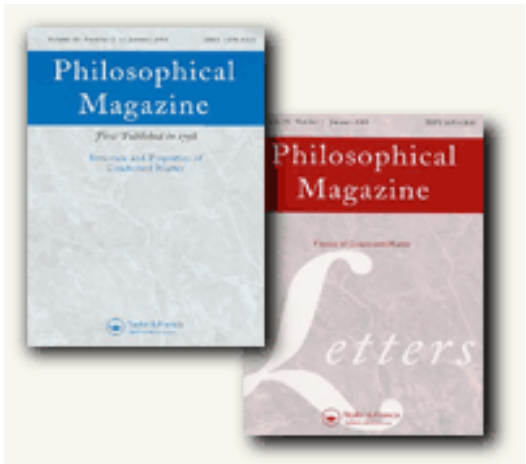

\section{A mean field theory for diffusion in a dilute multi- component alloy: a new model for the effect of solutes on self-diffusion}

\begin{tabular}{|r|l|}
\hline Journal: & Philosophical Magazine \& Philosophical Magazine Letters \\
\hline Manuscript ID: & TPHM-04-Dec-0137.R1 \\
\hline Journal Selection: & Philosophical Magazine \\
\hline Date Submitted by the \\
Author: & 30-May-2005 \\
\hline Komplete List of Authors: & Nastar, Maylise; CEA-Saclay, DMN/SRMP \\
\hline Keywords: & $\begin{array}{l}\text { transport properties, statistical physics, non-equilibrium } \\
\text { phenomena, metallic alloys, diffusion, atomistic simulation, atomic } \\
\text { transport, atomic defects }\end{array}$ \\
\hline Keywords (user supplied): & \multicolumn{2}{|c}{} \\
\hline
\end{tabular}

\section{(5) SholaroNE \\ Manuscript Central}




\title{
A mean field theory for diffusion in a dilute multi-component alloy : a new model for the effect of solutes on self-diffusion
}

\author{
Maylise Nastar* \\ Service de Recherches de Métallurgie Physique, CEA/Saclay, \\ 91191 Gif-sur-Yvette, France.
}

(Dated: May 30, 2005)

\begin{abstract}
A new extension of the self-consistent mean field (SCMF) theory is developed to describe diffusion in dilute alloys, special attention being paid to the problem of self-diffusion in presence of solute atoms. We start from a microscopic model of the atom-vacancy exchange frequency including nearest neighbour interactions and derive kinetic equations from a Master equation. The non-equilibrium distribution function is expressed trough time dependent effective interactions. Their range of interaction is controlling the level of description of the paths of a vacancy after a first exchange. In contrast to the previous diffusion models devoted to concentrated alloys, the present formulation makes appear into the final result several exchange frequencies associated to a given atom depending on the chemical species of the atoms nearby. A first approximation restricted to nearest neighbour effective interactions yields analytical expressions of the transport coefficients of a face centered cubic dilute binary alloy.

${ }^{*}$ Electronic address: mnastar@cea.fr
\end{abstract}


The phenomenological coefficients are equivalent to the ones obtained using the five frequency model within the first shell approximation. A new expression of the self-diffusion coefficient is proposed and compared to Monte carlo (MC) simulations using the same atomic diffusion model. The SCMF theory reproduces the main tendencies of the MC simulations, in particular within the random alloy region where the recent five-frequency model was not satisfying. The limitations and future improvements of the SCMF approach are easily related to the range of the effective interactions considered.

\section{INTRODUCTION}

Matter transport in alloys is generally controlled by microscopic jumps of point defects. In the limiting case of a very dilute binary alloy, the link between these microscopic jump frequencies and the phenomenological transport coefficients $L_{i j}$ has been established for the main crystallographic structures (Allnatt and Lidiard 1993). In that case it is possible after an exchange of a vacancy with a given atom to account for all the different vacancy paths and determine the probability for a vacancy to exchange again with the same atom. The five-frequency model for a dilute face centered cubic (fcc) alloy is a well-known example of this kind (Howard and Lidiard 1963, 1964 and Allnatt 1981 for the exact solution). But as soon as we consider the effect of adding a third atomic species or increasing the amount of solutes rigorous models are lacking. We present here the example of estimation of the diffusion coefficient of an isotope $A^{*}$ in a binary alloy $A B$ (solute $B$ in $A$ ) which remains a challenge. Even so the question is of a great practical interest since many experimental results are measurements of isotope diffusion coefficients. Within a multi-frequency model, the isotope diffusion coefficient, $D_{A^{*}}$, is a sum of several partial diffusion coefficients relative to the position of $A^{*}$ with 
respect to the solute $B$ for which the vacancy paths are performed within a cut-off radius. Following the approach of Howard and Manning (1967), Ishioka and Koiwa (1984) performed a more extensive calculation based on larger cut-off radius but some of their results were called into question from 1986 (Allnatt and Lidiard 1986). For example Allnatt and Lidiard (1986) have pointed out that $D_{A^{*}}$ should decrease with the $B$ solute concentration when the vacancy-solute exchange frequency is zero since then an addition of immobile solute atoms should slow down the diffusion of tracer solvent atoms $A^{*}$. A recent publication suggested a possible reason for these shortcomings by showing that the correlation effects between different vacancy encounters were not introduced properly (Szabó and Beke 2004). An alternative theoretical approach which was first developed to treat diffusion in concentrated alloys (Manning 1971, Moleko, Allnatt and Allnatt 1989, Nastar et al. 2000) was verified to be more reliable for the prediction of diffusion of isotope $A *$ in the limiting case of a 'random dilute alloy' $A B$. In a 'random alloy' first introduced by Manning (1971) a vacancy-atom exchange frequency, $\omega_{x}$ is taken to be characteristic of the atom $x$ but independent of the environment which implies no interaction between the atoms. Among the random lattice gas models, the predictions of Moleko, Allnatt and Allnatt (1989) were shown to be very close to recent Monte Carlo simulations (Belova and Murch 2003b).

In a fcc alloy with interactions, recent Monte Carlo simulations (Belova and Murch 2003a) have shown that both treatments of Howard and Manning (1967) and Ishioka and Koiwa (1984) have major shortcomings in describing self-diffusion in presence of solute atoms. There are very few alternatives to these multi-frequency models. A mean field approach like the Path Probability Method (PPM) is based on an atomic diffusion model which depends on the local environment (Sato and Kikuchi 
1983, Sato et al. 1985 correcting the first versions of Kikuchi and Sato 1969). But the statistical approximation used to average the matter flow under a gradient of chemical potential and formulate the $L_{i j}$ leads to averaged jump frequencies, each one representing an atomic species. A recent approach based on the same jump frequency model and starting from a Taylor series expansion in powers of time of the time correlation functions appearing in the $L_{i j}$ (Qin et al. 1998) employs the detailed balance principle to making appear a set of jump frequencies associated to a given chemical species. However, the latter principle is not sufficient to make appear rotational jumps of type $\omega_{1}$ from other jumps like the dissociative jump of type $\omega_{3}$ in a fcc alloy. Indeed both jump frequencies describe a jump leaving the same initial state and they differ by their saddle point position only so that they cannot be connected by a detailed balance equality. The empirical approach of Bakker and Stolwijk (Bakker 1979, Stolwijk 1981) also based on an averaged treatment of the jump frequencies leads to the same conclusions. Our previous work based on a SCMF theory yielded a full Onsager matrix from an atomic diffusion model (Nastar 2000) but the resulting $L_{i j}$ made appear a limited number of averaged jump frequencies too. A severe consequence is that non-diagonal phenomenological coefficients are automatically positive which means that for example a kinetic coupling inducing an inversion of the direction of an atomic flux would not be predicted by such models. Our purpose is to show that within a self-consistent mean field (SCMF) formalism one can make appear a set of jump frequencies which in the particular case of a dilute alloy is equivalent to the one defined by a multi-frequency model. The example of diffusion in a fcc binary dilute alloy (solute B in A) with first nearest neighbour interactions will be treated. It requires that at least five jump frequencies comes out of the present approach : the B-vacancy exchange frequency $\left(\omega_{2}\right)$ and the 
four different A-vacancy exchange frequencies : $\omega_{3}$ when B is nearest neighbour of vacancy, $\omega_{4}$ the reverse jump of $\omega_{3}, \omega_{1}$ when $\mathrm{B}$ is nearest neighbour of both species of the exchanging pair and $\omega_{0}$ for the other jumps (Howard and Lidiard 1963, 1964, Allnatt and Lidiard 1993). First objective will be to recover the formulae of the $L_{i j}$ of the five-frequency models and then to propose a new analytical model of the self-diffusion coefficient to first order in solute concentration. To check the validity of the later result, we perform Monte Carlo simulations based on the same atomic diffusion model with nearest neighbour interactions. We use a slightly different procedure than the one of Belova and Murch (2003a) : the atomic diffusion model is defined everywhere so that the present MC simulations do not prevent configurations in which solute atoms are in close proximity.

First section starts from an atomic diffusion model and derives kinetic equations by means of a master equation. Non-equilibrium averages are estimated using a non-equilibrium distribution function which is defined in terms of a time dependent effective hamiltonian. In the following sections the SCMF approach is applied to fcc alloys. Section III writes the kinetic equations for a $f c c$ alloy in the case of an effective hamiltonian restricted to nearest neighbour (nn) interactions and yields an analytical expression of the transport coefficients in the limit of a dilute alloy. The present theory is compared to the five-frequency models (Allnatt and Lidiard 1993). The extension to multi-component alloys being quite obvious, a new formulae is suggested for the tracer diffusion $D_{A^{*}}$ in $A B$. Section IV presents the atomic diffusion models and the conditions of MC simulations. Section V is a discussion of the results compared to the MC simulations and the previous models, first in the random alloy limit and second in alloys with interactions. 


\section{THE SCMF THEORY}

Within the theory of linear non-equilibrium thermodynamics, the $L_{i j}$ are defined as the constants coupling the fluxes to the external forces which will be here the gradients of chemical potentials. The evaluation of ensemble averages of a system subject to an external gradient of chemical potential requires to define a non-equilibrium distribution function. First part of this chapter provides with a general formulae of the non-equilibrium distribution function in terms of non-equilibrium effective interactions. We introduce then a Master Equation which expresses the fact that transitions between a given configuration and the others are controlled by the vacancy jump frequencies. We obtain then a series of kinetic equations which describe the time evolution of mean occupancies of resp. one chemical species on one site, two chemical species on a pair of sites, etc.. The $L_{i j}$ are identified from the fluxes which are deduced from the continuity equation applied to the time derivative of point averages. As a first approximation we assume the effective Hamiltonian contains pair effective interactions only. We deduce their expressions as a function of gradients of chemical potentials by applying the steady state conditions to the time derivative of the two-point averages. The resulting $L_{i j}$ are written as a function of equilibrium averages. Last part of the section is demonstrating that the Onsager reciprocal relation is verified.

\section{A. The non-equilibrium distribution function}

The alloy is represented by a system of interacting atoms and vacancies distributed on a rigid lattice. A configuration of the alloy is defined by a vector $\mathbf{n}$, the components of which are the occupation 
numbers of all species on all sites $\left\{n_{1}^{A}, n_{1}^{B}, \ldots, n_{1}^{v} ; n_{2}^{A}, n_{2}^{B}, \ldots, n_{2}^{v} ; \ldots\right\}$ such that $n_{i}^{a}=1$ if site is occupied by species $a$ and zero if else. The corresponding configurational Hamiltonian is noted $\widehat{H}$. Transitions between the different configurations of the system are controlled by exchanges between atoms and vacancies. The probability per time unit of a single jump of an atom $\alpha$ at a site $i$ into a vacancy $v$ at a site $j$ is noted $\widehat{w}_{i j}^{\alpha v}(\mathbf{n})$.

Changes of a configuration $\mathbf{n}$ are specified by the master equation,

$$
\frac{d \widehat{P}(\mathbf{n}, t)}{d t}=\sum_{\tilde{\mathbf{n}}}[\widehat{W}(\tilde{\mathbf{n}} \rightarrow \mathbf{n}) \widehat{P}(\tilde{\mathbf{n}}, t)-\widehat{W}(\mathbf{n} \rightarrow \tilde{\mathbf{n}}) \widehat{P}(\mathbf{n}, t)]
$$

where $\widehat{W}(\mathbf{n} \rightarrow \tilde{\mathbf{n}})$ is the transition probability from a configuration $\mathbf{n}$ to a configuration $\tilde{\mathbf{n}}$ per time unit : it is defined in terms of the microscopic frequencies controlling the diffusion of atoms. By construction of the microscopic jump frequencies which obey micro-reversibility, each term of the sum entering equation (1) is zero when $\widehat{P}$ is taken as the equilibrium distribution function $\widehat{P}_{0}$ :

$$
\widehat{P}_{0}(\mathbf{n})=\exp \left[\beta\left(\Omega_{0}+\sum_{\alpha} \mu_{\alpha} \sum_{i} n_{i}^{\alpha}-\widehat{H}\right)\right]
$$

Here $\beta=1 / T$ is the reciprocal temperature, $\Omega$ is the grand canonical potential found from the usual normalization condition $\sum_{\mathbf{n}} \widehat{P}_{0}(\mathbf{n})=1$, and $\mu_{\alpha}$ is the chemical potential of the atoms $\alpha$ relative to vacancies, i.e. the difference between the chemical potential of atoms $\alpha$ and of vacancies. Indeed, since we describe a system with a fixed number of sites $N_{s}$, the constraint on site $i, \sum_{a} n_{i}^{a}=1$, implies that there are only $\left(N_{c}-1\right)$ independent chemical potentials, where $N_{c}$ is the number of components in a many-component system ; for our convenience we choose chemical potentials of atoms relative to vacancy as independent ones.

[*] We mark functions depending on configuration by the 'hat' sign, e.g. $\widehat{H}$ stands for $\widehat{H}(\mathbf{n})$. 
Following Vaks (1996), the non-equilibrium distribution function entering equation (1) is expressed in terms of a time dependent effective hamiltonian $\widehat{h}(t)$ :

$$
\widehat{P}(\mathbf{n}, t)=\widehat{P}_{0}(\mathbf{n}) \widehat{P}_{1}(\mathbf{n}, t),
$$

where $\widehat{P}_{0}(\mathbf{n})$ is the equilibrium distribution function and $\widehat{P}_{1}(\mathbf{n}, t)=\exp \left\{\beta\left[\delta \Omega(t)+\sum_{\alpha, i} \delta \mu_{i}^{\alpha}(t) n_{i}^{\alpha}-\right.\right.$ $\widehat{h}(t)]\}$ is the non equilibrium contribution which is equal to one at equilibrium. The time-dependent effective Hamiltonian is written as a polynomial function of the occupation numbers

$$
\widehat{h}(t)=\frac{1}{2 !} \sum_{\alpha \beta, i \neq j} v^{(2)}{ }_{i j}^{\alpha \beta}(t) n_{i}^{\alpha} n_{j}^{\beta}+\frac{1}{3 !} \sum_{\alpha \beta \gamma, i \neq j \neq k} v_{i j k}^{(3)}{ }_{i j k}^{\alpha \beta \gamma}(t) n_{i}^{\alpha} n_{j}^{\beta} n_{k}^{\gamma}+\ldots
$$

where $v_{i j \ldots}^{(N)}{ }_{i j \ldots}^{\alpha \beta}(t)$ are time dependent $N$-body effective interactions, which are unknown and must be obtained solving the master equation. In fact, instead of searching $\widehat{P}(\mathbf{n}, t)$ as a solution of the master equation (1) we define $\widehat{P}$ by its moments, $\left\langle n_{i}^{\alpha}\right\rangle,\left\langle n_{i}^{\alpha} n_{j}^{\beta}\right\rangle, \ldots$, which we later call the one, two, ..., point averages, and establish the kinetic equations for the latter. Furthermore the non-equilibrium averages appearing in the kinetic equations are split up into two parts : a non-equilibrium contribution denoted by $\widehat{P}_{1}$ which is considered as a function to be averaged and the equilibrium average which is seen as the distribution function to be employed to calculating the ensemble average. It is convenient in doing so because the new averages to be calculated can now be connected to the well known thermodynamic statistical approximations. Two hierarchies of approximations come up at this point. First hierarchy is related to the statistical approximation used to calculate the equilibrium averages. The second level of approximation is associated with the number of unknown effective interactions of the time

[†] Referred to the "cluster expansion theorem" (Sanchez 1984), any quantity which is a function of configuration only can be written in the form of equation (4) 
dependent effective Hamiltonian that we consider. It determines the number of independent kinetic equations. For example truncation of the effective Hamiltonian after the pair effective interactions limits the system of independent kinetic equations to the point and two-point equations and the stationarity conditions are not guaranteed to be satisfied by the N-point kinetic equations $(N>2)$. Self-consistency is then guaranteed up to the two-point averages.

\section{B. Kinetic equations}

Starting from the master equation, the time derivative of such averages simply writes (demonstration is given in Appendix A of Nastar 2000),

$$
\begin{aligned}
\frac{d\left\langle n_{i}^{\alpha} n_{j}^{\beta} n_{k}^{\gamma} \ldots\right\rangle}{d t}= & \sum_{s \neq i \neq j \neq \ldots}\left\langle n_{j}^{\beta} n_{k}^{\gamma} \ldots\left[n_{s}^{\alpha} n_{i}^{v} \widehat{w}_{s i}^{\alpha v}-n_{i}^{\alpha} n_{s}^{v} \widehat{w}_{i s}^{\alpha v}\right]\right\rangle+ \\
& \sum_{s \neq i \neq j \neq \ldots}\left\langle n_{i}^{\alpha} n_{k}^{\gamma} \ldots\left[n_{s}^{\beta} n_{j}^{v} \widehat{w}_{s j}^{\beta v}-n_{j}^{\beta} n_{s}^{v} \widehat{w}_{j s}^{\beta v}\right]\right\rangle+\ldots,
\end{aligned}
$$

where the brackets indicate non-equilibrium ensemble averages. To calculate such averages we use two approximations : first we restrict the effective Hamiltonian to pair effective interactions, second we use a better statistical approximation than the Bragg Williams approximation used in (Nastar 2000). The complete procedure is presented below for a general model of alloy where the equilibrium averages are not explicitly calculated. Chapter (III) applies the model to an fcc dilute alloy and provides with a complete calculation of the $L_{i j}$ and the tracer correlation factor of a binary alloy.

After linearization with respect to the terms of the form $\beta\left(\delta \mu_{s}^{\alpha}-\delta \mu_{i}^{\alpha}\right)$ and $\beta \widehat{h}_{s}^{\alpha}$ together with the principle of detailed balance, we obtain for the 2-point average (details are given in appendix A) :

$$
\frac{d\left\langle n_{i}^{\alpha} n_{j}^{\beta}\right\rangle}{d t}=\beta \sum_{s \neq i \neq j \neq \ldots}\left\langle n_{j}^{\beta}\left[n_{s}^{\alpha} n_{i}^{v} \widehat{\omega}_{s i}^{\alpha v}\left(\delta \mu_{s}^{\alpha}-\delta \mu_{i}^{\alpha}-\widehat{h}_{s}^{\alpha}+\widehat{h}_{i}^{\alpha}\right)\right]\right\rangle^{(0)}+
$$




$$
\beta \sum_{s \neq i \neq j \neq \ldots}\left\langle n_{i}^{\alpha}\left[n_{s}^{\beta} n_{j}^{v} \widehat{\omega}_{s j}^{\beta v}\left(\delta \mu_{s}^{\beta}-\delta \mu_{j}^{\beta}-\widehat{h}_{s}^{\beta}+\widehat{h}_{j}^{\beta}\right)\right]\right\rangle^{(0)},
$$

where ${ }^{(0)}$ means averaging over the equilibrium distribution function $P^{(0)}$, and $\widehat{h}_{s}^{\alpha}$ is the partial derivative of $\widehat{h}$ with respect to $n_{s}^{\alpha} .\left(\delta \mu_{s}^{\beta}-\delta \mu_{j}^{\beta}\right)$ is equivalent to the difference of the total chemical potentials since the equilibrium contribution to the difference is equal to zero.

Flux of atomic species is deduced from the continuity equation applied to the kinetic equation of the one-point average :

$$
\frac{d\left\langle n_{i}^{\alpha}\right\rangle}{d t}=-\sum_{s \neq i} J_{i \rightarrow s}^{\alpha}
$$

and is recognized to be :

$$
J_{i \rightarrow s}^{\alpha}=-\beta\left[\left\langle n_{i}^{\alpha} n_{s}^{v} \widehat{\omega}_{i s}^{\alpha v}\left(\mu_{s}^{\alpha}-\mu_{i}^{\alpha}-\widehat{h}_{s}^{\alpha}+\widehat{h}_{i}^{\alpha}\right)\right\rangle^{(0)} .\right.
$$

Following the same procedure presented in (Nastar 2000), we could show that close to an homogeneous equilibrium and under an homogeneous gradient of chemical potential, flux $J_{i \rightarrow j}^{\alpha}$ and $\frac{d\left\langle n_{i}^{\alpha} n_{j}^{\beta}\right\rangle}{d t}-\frac{d\left\langle n_{j}^{\alpha} n_{i}^{\beta}\right\rangle}{d t}$ depends on asymmetrical effective interactions of type $\left(v_{i j}^{\alpha \beta}-v_{j i}^{\alpha \beta}\right)$. An easy way to account for this property is to put equal to zero the symmetric part of the effective interactions and assume $v_{i j}^{\alpha \beta}=-v_{j i}^{\alpha \beta}$ and $v_{i j}^{\alpha \alpha}=0$. In an isotope crystal, the diffusion problem is mono-dimensional : we choose an axis along which the atomic fluxes are estimated. This axis is an axis of rotational symmetry so that we group rotationally equivalent sites together. Furthermore, in an homogeneous equilibrium state, the local concentrations do not depend on sites : $c_{i}^{\alpha}=c_{\alpha}$, where $c_{\alpha}$ is the composition of atoms $\alpha$. For such conditions the system of equations has translational symmetry ; nothing is changed under the transformation $r \rightarrow r+R$ (where $R$ is a vector between two arbitrary sites of the lattice). The solution may be taken with the same symmetry : $v_{i j}^{\alpha \beta}=v_{\alpha \beta}\left(r_{i}+R, r_{j}+R\right)$. Therefore, the asymmetrical 
property added to the translation property reduce the number of unknown effective interactions to the number of non-equivalent two-point averages to be considered. Each non-equivalent pair of points is labelled by a number $i_{v}\left(i_{v}=1\right.$ for the first nearest neighbour two-point average) and is associated to a positive effective asymmetrical interaction : $v_{(\alpha \sigma)^{i v}}=v_{\left(i i^{+}\right)^{i v}}^{\alpha \sigma}$ in which $\left(i i^{+}\right)^{i_{v}}$ is the axis coordinate of the vector linking both sites.

The antisymmetric property of $v$ leads to a simplification of the flux :

$$
J_{i \rightarrow i^{+}}^{\alpha}=-\beta\left[\left\langle n_{i}^{\alpha} n_{i^{+}}^{v} \widehat{\omega}_{i i^{+}}^{\alpha v}\left(\mu_{i^{+}}^{\alpha}-\mu_{i}^{\alpha}+2 h_{i}^{\alpha}\right)\right\rangle^{(0)} .\right.
$$

Since the effective Hamiltonian contains pair interactions only we write down the flux in terms of the $v_{(\alpha \sigma)^{i v}}:$

$$
J_{i \rightarrow i^{+}}^{\alpha}=-\beta l_{\alpha}^{(0)}\left(\mu_{i^{+}}^{\alpha}-\mu_{i}^{\alpha}\right)-\beta \sum_{\sigma, i_{v}}\left[l_{\alpha}\left(\sigma, i_{v}\right) v_{(\alpha \sigma)^{i v}}\right]
$$

with

$$
l_{\alpha}^{(0)}=\gamma_{\left(i i^{+}\right)^{1}}\left\langle n_{i}^{\alpha} n_{i^{+}}^{v} \widehat{\omega}_{i i^{+}}^{\alpha v}\right\rangle^{(0)}
$$

and

$$
l_{\alpha}\left(\sigma, i_{v}\right)=2 \sum_{p \neq i \neq i^{+}, \sigma} \gamma_{\left(i i^{+}\right)^{1}} \gamma_{(i p)^{i_{v}}}\left\langle n_{i}^{\alpha} n_{i^{+}}^{v} n_{p}^{\sigma} \widehat{\omega}_{i i^{+}}^{\alpha v}\right\rangle^{(0)} \frac{v_{i p}^{\alpha \sigma}}{v_{(\alpha \sigma)}^{i_{v}}} .
$$

$\gamma_{(i p)^{i_{v}}}$ is equal to 1 if sites $i$ and $p$ form a pair of type $i_{v}$ and is equal to 0 if else.

The phenomenological coefficients are by definition the coefficients which relate fluxes to thermodynamic forces (here gradients of chemical potentials). They have the form :

$$
1 / \beta L_{\alpha \alpha}=l_{\alpha}^{(0)}(\alpha)+\sum_{\sigma, i_{v}} l_{\alpha}\left(\sigma, i_{v}\right) v_{(\alpha \sigma)^{i v}}(\alpha)
$$




$$
1 / \beta L_{\alpha \beta}=\sum_{\sigma, i_{v}} l_{\alpha}\left(\sigma, i_{v}\right) v_{(\alpha \sigma)^{i v}}(\beta),
$$

where $v_{(\alpha \beta)^{i_{v}}}(\sigma)$ is the $\sigma$ coordinate of $v_{(\alpha \beta)^{i_{v}}}: v_{(\alpha \beta)^{i_{v}}}=\sum_{\sigma} v_{(\alpha \beta)^{i v}}(\sigma)\left(\nabla \mu_{\sigma}\right)$, with $\left(\nabla \mu_{\sigma}\right)=$ $\gamma_{\left(i i^{+}\right)^{1}}\left(\mu_{i^{+}}^{\sigma}-\mu_{i}^{\sigma}\right)$. In setting down equation (10) we have omitted the time dependence of $v$ since the kinetic equations will be solved under steady state conditions. The two point kinetic equations alone are required for the estimation of $v$. They can be written as :

$$
\begin{aligned}
& 1 / \beta \frac{d\left\langle n_{i}^{\alpha} n_{j}^{\beta}\right\rangle^{\left(i_{v}\right)}}{d t}=m_{\alpha}\left(\beta, i_{v}\right) \nabla \mu_{\alpha}-m_{\beta}\left(\alpha, i_{v}\right) \nabla \mu_{\beta} \\
& +\sum_{j_{v}}\left[t_{(\alpha \beta)^{i} v,(\alpha \beta)^{j_{v}}} v_{(\alpha \beta)^{j_{v}}}+\sum_{\gamma} t_{(\alpha \beta)^{i_{v}},(\alpha \gamma)^{j_{v}}} v_{(\alpha \gamma)^{j_{v}}}+\sum_{\gamma} t_{(\alpha \beta)^{i_{v}},(\gamma \beta)^{j_{v}}} v_{(\gamma \beta)^{j_{v}}}\right]
\end{aligned}
$$

with

$$
\begin{aligned}
& m_{\sigma}\left(\alpha, i_{v}\right)=\sum_{s \neq i \neq i^{+}} \gamma_{\left(i i^{+}\right)^{i} v} \gamma_{(i s)^{1}}\left\langle n_{i^{+}}^{\sigma} n_{s}^{\alpha} n_{i}^{v} \widehat{\omega}_{s i}^{\sigma v}\right\rangle^{(0)}\left(\mu_{s}^{\sigma}-\mu_{i}^{\sigma}\right) / \nabla \mu_{\sigma} \\
& t_{(\alpha \beta)^{i v},(\alpha \sigma)^{j_{v}}}=\left[\sum_{s \neq i \neq i^{+}} \gamma_{\left(i i^{+}\right)^{i_{v}}} \gamma_{(i s)^{1}}\left\langle n_{i^{+}}^{\beta} n_{s}^{\alpha} n_{i}^{v} \widehat{\omega}_{s i}^{\alpha v}\left(\sum_{p \neq j, s} \gamma_{(i p)^{j v}} v_{i p}^{\alpha \sigma} n_{p}^{\sigma}-\sum_{p \neq i, i^{+}} \gamma_{(s p)^{j v}} v_{s p}^{\alpha \sigma} n_{p}^{\sigma}\right)\right\rangle^{(0)}\right] / v_{(\alpha \sigma)^{j v}}(17) \\
& \text { and } \\
& t_{(\alpha \beta)^{i v},(\alpha \beta)^{j_{v}}}=t_{(\alpha \beta)^{i_{v}},(\alpha \sigma)^{j_{v}}}(\sigma \equiv \beta)+t_{(\alpha \beta)^{i v},(\sigma \beta)^{j_{v}}}(\sigma \equiv \alpha) \\
& +\left[\gamma_{(i j)^{i v}} \sum_{s \neq i \neq j} \gamma_{(i s)^{1}}\left\langle n_{j}^{\beta} n_{s}^{\alpha} n_{i}^{v} \widehat{\omega}_{s i}^{\alpha v}\left(\gamma_{(i j)^{j} v} v_{i j}^{\alpha \beta}-\gamma_{(s j)^{j v}} v_{s j}^{\alpha \beta}\right)\right\rangle\right. \\
& \left.+\gamma_{(i j)^{i v}} \sum_{s \neq i \neq j} \gamma_{(j s)^{1}}\left\langle n_{i}^{\alpha} n_{s}^{\beta} n_{j}^{v} \widehat{\omega}_{s j}^{\beta v}\left(\gamma_{(i j)^{j v}} v_{i j}^{\alpha \beta}-\gamma_{(s i)^{j v}} v_{s i}^{\beta \alpha}\right)\right\rangle^{(0)}\right] / v_{(\alpha \beta)^{j v}} .
\end{aligned}
$$

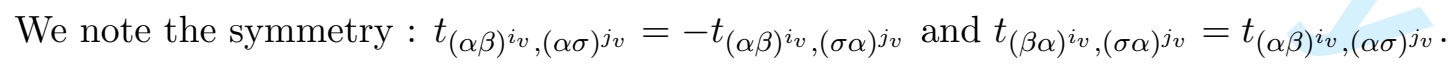


The unknown effective interactions are then solution of equation (15) under steady state conditions (derivatives with time are equal to zero). They are given as a linear combination of the gradients of chemical potential :

$$
\overline{\bar{T}} \bar{v}=\bar{b}
$$

Components of $\bar{b}$ and $\bar{v}, b(i)$ and $v(i)$, are associated to the couple $(\alpha, \sigma)^{i_{v}}$ with $i=\left(N_{e s p} *\left(N_{e s p}+\right.\right.$ $1) / 2) *\left(i_{v}-1\right)+N_{e s p}(\alpha-1)+\sigma . b(i)$ is equal to $-m_{\alpha}\left(\sigma, i_{v}\right) \nabla \mu_{\alpha}+m_{\sigma}\left(\alpha, i_{v}\right) \nabla \mu_{\sigma}$. Element $t(i, j)$ of matrix $\mathrm{T}$ is associated to the couple $\left((\alpha \sigma)^{i v},(\beta \gamma)^{j v}\right)$ such that $i$ is the index defined above and $j=\left(N_{e s p} *\left(N_{e s p}+1\right) / 2\right) *\left(j_{v}-1\right)+N_{e s p}(\beta-1)+\gamma$.

\section{The Onsager reciprocal relation}

The SCMF approximation limited to nn effective interactions is shown to satisfy the Onsager reciprocal relation, that is, $L_{\alpha \beta}=L_{\beta \alpha}$.

For that it is sufficient to note that

$$
l_{\alpha}(\sigma, 1)=2 m_{\alpha}(\sigma, 1) \text {. }
$$

Using this relationship we deduce $L_{\alpha \beta}$ from equation (14) where the unknown $v_{(\alpha \sigma)^{1}}(\beta)$ are calculated using the system of equations (15) :

$$
\begin{aligned}
L_{\alpha \beta}= & +2 \sum_{\sigma>\alpha} m_{\alpha}(\sigma, 1) t_{(\alpha \sigma)^{1}(\alpha \beta)^{1}}^{-1} m_{\beta}(\alpha, 1) \\
& +2 \sum_{\sigma>\beta} m_{\alpha}(\sigma, 1) t_{(\alpha \sigma)^{1}(\beta \sigma)^{1}}^{-1} m_{\beta}(\sigma, 1) \\
& +2 \sum_{\sigma>\beta} m_{\alpha}(\beta, 1) t_{(\alpha \beta)^{1}(\beta \sigma)^{1}}^{-1} m_{\beta}(\sigma, 1),
\end{aligned}
$$


where $t_{(\alpha \sigma)^{1}(\alpha \beta)^{1}}^{-1}$ is the element of the inverse $T$ matrix located at the arrow associated to $(\alpha \sigma)^{1}$ and the column associated to $(\alpha \beta)^{1}$. In the same way, $L_{\beta \alpha}$ is deduced from equation (14) by exchanging $\alpha$ and $\beta$ where the unknown $v_{(\beta \alpha)^{1}}(\alpha)$ are calculated using the system of equations (15) :

$$
\begin{aligned}
L_{\beta \alpha}= & +2 \sum_{\sigma>\beta} m_{\beta}(\sigma, 1) t_{(\beta \sigma)^{1}(\alpha \beta)^{1}}^{-1} m_{\alpha}(\beta, 1) \\
& +2 \sum_{\sigma>\alpha} m_{\beta}(\alpha, 1) t_{(\alpha \beta)^{1}(\alpha \sigma)^{1}}^{-1} m_{\alpha}(\sigma, 1) \\
& \left.+2 \sum_{\sigma>\beta} m_{\beta}(\sigma, 1) t_{(\beta \sigma)^{1}(\alpha \sigma)^{1}}^{-1} m_{\alpha}(\sigma, 1)\right] ;
\end{aligned}
$$

which leads to the equality: $L_{\beta \alpha}=L_{\alpha \beta}$.

\section{RESULTS}

A. The fcc alloy

The preceding section has been concerned with the general expression of the $L_{i j}$. To complete the derivation, the crystallographic structure needs to be specified. Indeed an explicit expression of the sums over neighbouring sites entering equations $(16,17,18)$ will depend on the crystal. In the following sections we will illustrate the theory on the fcc crystallographic structure. Moreover, for the sake of simplicity and in order to keep analytical results, we make the approximation that the effective Hamiltonian is restricted to nearest neighbour $(\mathrm{nn})$ interactions. Most of this section takes advantage of the homogeneity of the equilibrium state. In the dilute limit, one can show that N-point averages are equal to the product of point averages multiplied by an exponential of a binding energy so that they are characterized by their interacting bonds only and disconnected from the sites surrounding the N-point cluster. In the particular case of an Hamiltonian limited to nn interactions, the N-point 
averages are characterized by their nn pairs only. It is no more valid in concentrated alloys but we will make this assumption from the beginning since the kinetic equations will be later used in a fcc dilute alloy. There are two steps in the derivation. First step is to identify the N-point averages to be calculated and last step is to calculate them. Sums over neighbours entering the kinetic equations are calculated along a [100] symmetry axis. In practice, $l_{\alpha}^{(0)}$ of equation (11) may be written as

$$
l_{\alpha}^{(0)}=\gamma_{(\alpha v)^{1}}\left\langle n_{\alpha} n_{v} \widehat{\omega}_{\alpha v}\right\rangle^{(0)},
$$

where indices entering equation (11) are eliminated since equilibrium averages are characterized by their first nearest neighbour bonds only and $\gamma_{(\alpha v)^{1}}$ function is introduced to notify that species $\alpha$ and $v$ occupy nn sites. In the same way equation (16) reduces to :

$$
m_{\sigma}(\alpha, 1)=-3 \gamma_{(\sigma v)^{1}} \gamma_{(\alpha \sigma)^{1}}\left\langle n_{\alpha} n_{\sigma} n_{v} \widehat{\omega}_{\sigma v}\right\rangle^{(0)}+2 \gamma_{(\alpha v)^{1}} \gamma_{(\alpha \sigma)^{1}} \gamma_{(\sigma v)^{1}}\left\langle n_{\alpha} n_{\sigma} n_{v} \widehat{w}_{\sigma v}\right\rangle^{(0)}
$$

where summations over neighbours entering equation (16) are performed along the [100] axis.

We start with a pair of sites $\left(i i^{+}\right)^{1}$ located at the respective positions $i$ and $i+1$ along the [100] axis. Among the nn of $i$, there is one site at position $(i+1)$ which does not coincide with $i^{+}$and is not $\mathrm{nn}$ of $i^{+}$and there are four sites at position $(i-1)$ which are not $\mathrm{nn}$ of $i^{+}$, which leads to the factor $(1-4=-3)$ in front of the first average of equation (24). Furthermore there are two sites at position $(i+1)$ which are nn of both $s$ and $i$ which explains the factor (2) in front of the second average.

Since nn neighbour effective interactions are considered only, terms of type $t_{(\alpha \beta)^{1},(\alpha \gamma)^{1}}$ are to retain only. They involve the calculation of four-point averages. The counting of these sums in a fcc crystal is presented in appendix $\mathrm{B}$. 


$$
\begin{aligned}
& t_{(\alpha \beta)^{1},(\alpha \sigma)^{1}}=-8 \gamma_{(\alpha \beta)^{1}} \gamma_{(\alpha v)^{1}} \gamma_{(\alpha \sigma)^{1}}\left\langle\widehat{\omega}_{\alpha v} n_{\alpha} n_{\beta} n_{v} n_{\sigma}\right\rangle^{(0)} \\
& -10 \gamma_{(\alpha \beta)^{1}} \gamma_{(\alpha v)^{1}} \gamma_{(\alpha \sigma)^{1}} \gamma_{(\sigma v)^{1}}\left\langle\widehat{\omega}_{\alpha v} n_{\alpha} n_{\beta} n_{v} n_{\sigma}\right\rangle^{(0)} \\
& +10 \gamma_{(\alpha \beta)^{1}} \gamma_{(\alpha v)^{1}} \gamma_{(\alpha \sigma)^{1}} \gamma_{(\sigma \beta)^{1}}\left\langle\widehat{\omega}_{\alpha v} n_{\alpha} n_{\beta} n_{v} n_{\sigma}\right\rangle^{(0)}+11 \gamma_{(\alpha \beta)^{1}} \gamma_{(\alpha v)^{1}} \gamma_{(\sigma v)^{1}}\left\langle\widehat{\omega}_{\alpha v} n_{\alpha} n_{\beta} n_{v} n_{\sigma}\right\rangle^{(0)} \\
& -2 \gamma_{(\alpha \beta)^{1}} \gamma_{(\alpha v)^{1}} \gamma_{(\sigma v)^{1}} \gamma_{(\beta \sigma)^{1}}\left\langle\widehat{\omega}_{\alpha v} n_{\alpha} n_{\beta} n_{v} n_{\sigma}\right\rangle^{(0)}-2 \gamma_{(\alpha \beta)^{1}} \gamma_{(\alpha v)^{1}} \gamma_{(\sigma v)^{1}} \gamma_{(\beta \sigma)^{1}} \gamma_{(\alpha \sigma)^{1}}\left\langle\widehat{\omega}_{\alpha v} n_{\alpha} n_{\beta} n_{v} n_{\sigma}\right\rangle^{(0)} \\
& -10 \gamma_{(\alpha \beta)^{1}} \gamma_{(\alpha v)^{1}} \gamma_{(\beta v)^{1}} \gamma_{(\alpha \sigma)^{1}}\left\langle\widehat{\omega}_{\alpha v} n_{\alpha} n_{\beta} n_{v} n_{\sigma}\right\rangle^{(0)}+4 \gamma_{(\alpha \beta)^{1}} \gamma_{(\alpha v)^{1}} \gamma_{(\beta v)^{1}} \gamma_{(\alpha \sigma)^{1}} \gamma_{(\sigma v)^{1}}\left\langle\widehat{\omega}_{\alpha v} n_{\alpha} n_{\beta} n_{v} n_{\sigma}\right\rangle^{(0)} \\
& +4 \gamma_{(\alpha \beta)^{1}} \gamma_{(\alpha v)^{1}} \gamma_{(\beta v)^{1}} \gamma_{(\alpha \sigma)^{1}} \gamma_{(\beta \sigma)^{1}}\left\langle\widehat{\omega}_{\alpha v} n_{\alpha} n_{\beta} n_{v} n_{\sigma}\right\rangle^{(0)} \\
& +2 \gamma_{(\alpha \beta)^{1}} \gamma_{(\alpha v)^{1}} \gamma_{(\beta v)^{1}} \gamma_{(\alpha \sigma)^{1}} \gamma_{(\beta \sigma)^{1}} \gamma_{(\sigma v)^{1}}\left\langle\widehat{\omega}_{\alpha v} n_{\alpha} n_{\beta} n_{v} n_{\sigma}\right\rangle^{(0)} \\
& -6 \gamma_{(\alpha \beta)^{1}} \gamma_{(\alpha v)^{1}} \gamma_{(\beta v)^{1}} \gamma_{(\beta \sigma)^{1}} \gamma_{(\sigma v)^{1}}\left\langle\widehat{\omega}_{\alpha v} n_{\alpha} n_{\beta} n_{v} n_{\sigma}\right\rangle^{(0)} . \\
& t_{(\alpha \beta)^{1},(\alpha \beta)^{1}}=t_{(\alpha \beta)^{1},(\alpha \sigma)^{1}}(\sigma \equiv \beta)+t_{(\beta \alpha)^{1},(\beta \sigma)^{1}}(\sigma \equiv \alpha) \\
& +7 \gamma_{(\alpha \beta)^{1}} \gamma_{(\alpha v)^{1}}\left\langle\widehat{\omega}_{\alpha v} n_{\alpha} n_{\beta} n_{v}\right\rangle^{(0)}+2 \gamma_{(\alpha \beta)^{1}} \gamma_{(\alpha v)^{1}} \gamma_{(\beta v)^{1}}\left\langle\widehat{\omega}_{\alpha v} n_{\alpha} n_{\beta} n_{v}\right\rangle^{(0)} \\
& +7 \gamma_{(\alpha \beta)^{1}} \gamma_{(\beta v)^{1}}\left\langle\widehat{\omega}_{\beta v} n_{\alpha} n_{\beta} n_{v}\right\rangle^{(0)}+2 \gamma_{(\alpha \beta)^{1}} \gamma_{(\alpha v)^{1}} \gamma_{(\beta v)^{1}}\left\langle\widehat{\omega}_{\beta v} n_{\alpha} n_{\beta} n_{v}\right\rangle^{(0)}
\end{aligned}
$$

In principle this model is valid at any concentration of the alloy. Difficulties arise when one decides to calculate the equilibrium averages. Such averages should respect consistency between thermodynamics and kinetics and in particular should satisfy the detailed balance,

$$
\left\langle n_{i}^{\alpha} n_{s}^{v} \widehat{\omega}_{i s}^{\alpha v}\right\rangle^{(0)}=\left\langle n_{s}^{\alpha} n_{i}^{v} \widehat{\omega}_{s i}^{\alpha v}\right\rangle^{(0)} .
$$

This requirement is easy to satisfy in a dilute alloy at first order in the solute concentration. Because 
the averaged terms are written so as to make appear the exact atomic jump frequencies which by construction respect the detailed balance.

\section{B. The dilute fcc alloy}

We turn now to the presentation of explicit expressions for the transport coefficients in a binary dilute alloy. A dilute alloy is an easy case since thermodynamic averages of product of occupation numbers are known. Starting from the classical rate theory, we adopt an exponential form for the jump frequencies which depends upon the configuration of nearby atoms and defects. Furthermore we make the assumption that local configuration is limited to the first coordination shell.

It requires to calculate the probability of every shell configuration and to allocate to it the corresponding value of the jump frequency. For example :

$$
\begin{array}{r}
l_{A}^{(0)}=\gamma_{(i s)^{1}}\left\langle n_{i}^{\alpha} n_{s}^{v} \widehat{\omega}_{i s}^{A v}\right\rangle^{(0)} \\
=\sum_{\sigma_{i^{\prime}}, \sigma_{s^{\prime}}, \sigma_{k}} \gamma_{(i s)^{1}}\left\langle n_{i}^{\alpha} n_{s}^{v} n_{i_{1}^{\prime}}^{\sigma_{1}} \ldots n_{i_{7}^{\prime}}^{\sigma_{7}} n_{s_{1}^{\prime}}^{\sigma_{1}} \ldots n_{s_{7}^{\prime}}^{\sigma_{7}} n_{k_{1}}^{\sigma_{1}} \ldots n_{k_{4}}^{\sigma_{4}} \widehat{\omega}_{i s}^{A v}\right\rangle^{(0)} \\
=\sum_{\sigma_{i^{\prime}}, \sigma_{s^{\prime}}, \sigma_{k}} \gamma_{(i s)^{1}}\left\langle n_{i}^{\alpha} n_{s}^{v} n_{i_{1}^{\prime}}^{\sigma_{1}} \ldots n_{i_{7}^{\prime}}^{\sigma_{7}} n_{s_{1}^{\prime}}^{\sigma_{1}} \ldots n_{s_{7}^{\prime}}^{\sigma_{7}} n_{k_{1}}^{\sigma_{1}} \ldots n_{k_{4}}^{\sigma_{4}}\right\rangle^{(0)} \omega_{A v}\left(n_{i_{1}^{\prime}}^{\sigma_{1}}=\ldots n_{s_{1}^{\prime}}^{\sigma_{1}}=\ldots n_{k_{1}}^{\sigma_{1}}=\ldots=1\right) .
\end{array}
$$

We introduce three types of sites : four sites of type $k$ which are nn of site $i$ and $s$, and seven sites of type $i^{\prime}\left(\right.$ resp. $\left.s^{\prime}\right)$ which are nn of site $i$ (resp. $s$ ). In a binary alloy, since the value of a jump frequency is fixed by the number of $B$ atoms which are on sites of the first shell no matter the interactions between them, it is then convenient to define jump frequencies by using the notation : $\omega_{A v}\left(\sigma_{i^{\prime}}, \sigma_{s^{\prime}}, \sigma_{k}\right)$ where $\sigma_{i^{\prime}}$ (resp. $\sigma_{s^{\prime}}, \sigma_{k}$ ) is the number of $B$ atoms on sites of type $i^{\prime}$ (resp. $s^{\prime}, k$ ). In the limit of low concentration, the first coordination shell contains at maximum one site occupied by a solute atom 
while the other sites are occupied by solvent atoms. As a consequence, averages make appear only one binding energy between solute and vacancy. It is easy to show that starting from CVM or low temperature expansion (Ducastelle 1991)

$$
\left\langle n_{i}^{v} n_{i^{\prime}}^{B}\right\rangle^{(0)} \simeq c_{B} c_{v} y_{B v}
$$

where $y_{B v}$ is the probability of forming a pair $B v$ which is an exponential of thermodynamic interactions extracted from $H$. Since jump frequencies satisfy the detailed balance principle, we get

$$
\gamma_{(A v)^{1}} \gamma_{(B v)^{1}}\left\langle n_{B} n_{A} n_{v} \omega_{A v}\right\rangle=\gamma_{(A v)^{1}} \gamma_{(A B)^{1}}\left\langle n_{B} n_{A} n_{v} \omega_{A v}\right\rangle
$$

A projection on the first shell coordination gives

$$
\begin{gathered}
\gamma_{(i s)^{1}}\left\langle n_{i}^{A} n_{s}^{v} n_{i_{1}^{\prime}}^{A} \ldots n_{i_{7}^{\prime}}^{A} n_{s_{1}^{\prime}}^{B} n_{s_{2}^{\prime}}^{A} \ldots n_{s_{7}^{\prime}}^{A} n_{k_{1}}^{A} \ldots n_{k_{4}}^{A}\right\rangle^{(0)} \omega_{A v}(0,1,0)= \\
\gamma_{(i s)^{1}}\left\langle n_{i}^{A} n_{s}^{v} n_{i_{1}^{\prime}}^{B} n_{i_{2}^{\prime}}^{A} \ldots n_{i_{7}^{\prime}}^{A} n_{s_{1}^{\prime}}^{A} \ldots n_{s_{7}^{\prime}}^{A} n_{k_{1}}^{A} \ldots n_{k_{4}}^{A}\right\rangle^{(0)} \omega_{A v}(1,0,0),
\end{gathered}
$$

which to first order in solute concentration, is equivalent to :

$$
c_{A}^{19} c_{v} c_{B} y_{B v} \omega_{A v}(0,1,0)=c_{A}^{19} c_{v} c_{B} \omega_{A v}(1,0,0)
$$

where $\omega_{A v}(0,1,0)$ (resp. $\left.\omega_{A v}(1,0,0)\right)$ corresponds to the so-called $\omega_{A v}^{3}\left(\right.$ resp. $\left.\omega_{A v}^{4}\right)$ of the five frequency model (Allnatt and Lidiard 1993). Hence the detailed balance gives the well known relationship : $y_{B v}=\omega_{A v}^{4} / \omega_{A v}^{3}$.

If we come back to equation(28) of $l_{A}^{(0)}$ we make appear two additional frequencies, $\omega_{A v}(0,0,0)$ and $\omega_{A v}(0,0,1)$ which are recognized to be the so-called $\omega_{A v}^{0}$ and $\omega_{A v}^{1}$ of the five frequency model :

$$
\begin{array}{r}
l_{A}^{(0)} \simeq c_{v}\left[c_{A}^{19} \omega_{A v}^{0}+7 c_{B} c_{A}^{18} \omega_{A v}^{4}+4 c_{B} c_{A}^{18} y_{B v} \omega_{A v}^{1}+7 c_{B} c_{A}^{18} y_{B v} \omega_{A v}^{3}\right. \\
\simeq c_{v} \omega_{A v}^{0}\left[1-c_{B}\left(19-14 \frac{\omega_{A v}^{4}}{\omega_{A v}^{0}}-4 \frac{\omega_{A v}^{1}}{\omega_{A v}^{0}} \frac{\omega_{A v}^{4}}{\omega_{A v}^{3}}\right)\right] .
\end{array}
$$


The above calculations can be easily extended to any averaged quantities. Let us first proceed with the derivation of the $L_{i j}$ in a binary alloy $A B$. When the effective interactions are limited to the nn ones, the associated matrix $T$ is a scalar equal to $t_{(A B)^{1}(A B)^{1}}$ and the $L_{i j}$ are deduced from equation $(21):$

$$
\begin{aligned}
& L_{A A}=l_{A}^{(0)}-2\left(m_{A}(B, 1)\right)^{2} / t_{(A B)^{1}(A B)^{1}} \\
& L_{A B}=2 m_{A}(B, 1) m_{B}(A, 1) / t_{(A B)^{1}(A B)^{1}} \\
& L_{B B}=l_{B}^{(0)}-2\left(m_{B}(A, 1)\right)^{2} / t_{(A B)^{1}(A B)^{1}}
\end{aligned}
$$

Following the procedure explained in equation (28), we express

$$
l_{B}^{(0)} \simeq c_{v} c_{B} \omega_{B v} \frac{\omega_{A v}^{4}}{\omega_{A v}^{3}}
$$

where $\omega_{B v}$ is the jump frequency of $B$ in pure $A$. We do not introduce the effect of surrounding $B$ atoms on $\omega_{B v}$ since $B$ concentration is supposed to be very low. In the same way,

$$
\begin{gathered}
m_{B}(A, 1)=-c_{v} c_{B} \omega_{B v} \frac{\omega_{A v}^{4}}{\omega_{A v}^{3}}, \\
m_{A}(B, 1)=c_{v} c_{B} \frac{\omega_{A v}^{4}}{\omega_{A v}^{3}}\left(2 \omega_{A v}^{1}-3 \omega_{A v}^{3}\right),
\end{gathered}
$$

and

$$
t_{(A B)^{1},(A B)^{1}}=c_{v} c_{B} \frac{\omega_{A v}^{4}}{\omega_{A v}^{3}}\left(2 \omega_{A v}^{1}+7 \omega_{A v}^{3}+2 \omega_{B v}\right) .
$$

Therefore, the resulting expressions for $L_{A A}, L_{A B}$ and $L_{B B}$ are :

$$
L_{A B}=2 \omega_{B v} c_{v} c_{B} \frac{\omega_{A v}^{4}}{\omega_{A v}^{3}} \frac{-2 \omega_{A v}^{1}+3 \omega_{A v}^{3}}{2 \omega_{A v}^{1}+7 \omega_{A v}^{3}+2 \omega_{B v}},
$$




$$
\begin{gathered}
L_{B B}=l_{B}^{(0)}-2 \omega_{B v} c_{v} c_{B} \frac{\omega_{A v}^{4}}{\omega_{A v}^{3}} \frac{\omega_{B v}}{2 \omega_{A v}^{1}+7 \omega_{A v}^{3}+2 \omega_{B v}}, \\
L_{A A}=l_{A}^{(0)}-2 c_{v} c_{B} \frac{\omega_{A v}^{4}}{\omega_{A v}^{3}} \frac{\left(3 \omega_{A v}^{3}-2 \omega_{A v}^{1}\right)^{2}}{2 \omega_{A v}^{1}+7 \omega_{A v}^{3}+2 \omega_{B v}},
\end{gathered}
$$

where $l_{A}^{(0)}$ is given by equation (33) and $l_{B}^{(0)}$ by equation (37). These transport coefficients turn out to be the same as those obtained by Howard and Lidiard $(1963,1964)$ using the five frequency model within the first shell approximation. Taking into account nn effective interactions only would mean calculating the probability of the direct jumping back of a vacancy in competition with the escaping jumps, the paths of the vacancy made of more than two jumps being neglected.

We turn now to the evaluation of the self-diffusion coefficient. To do so we add to the binary alloy $A B$ a third atomic species, an isotope $A^{*}$ of solvent $A$, and calculate the appropriate transport coefficient $L_{A^{*} A^{*}}:$

$$
D_{A^{*}}=1 / \beta \lim _{c_{A^{*}} \rightarrow 0} L_{A^{*} A^{*}}
$$

Following the notation of equation (13) we write down $L_{A^{*} A^{*}}$ :

$$
\begin{array}{r}
L_{A * A *} / \beta=l_{A *}^{(0)}-l_{A *}(A, 1) v_{A A *}(A *)-l_{A *}(B, 1) v_{B A *}(A *) \\
=l_{A *}^{(0)}+2 \frac{m_{A *}(A, 1) t_{(A B)^{1}(A B)^{1}}}{\operatorname{det}(T)}\left[-m_{A *}(A, 1) t_{(B A *)^{1}(B A *)^{1}}+m_{A *}(B, 1) t_{A A *, B A *}\right] \\
+2 \frac{m_{A *}(B, 1) t_{(A B)^{1}(A B)^{1}}}{\operatorname{det}(T)}\left[m_{A *}(A, 1) t_{(B A *)^{1}(A A *)^{1}}-m_{A *}(B, 1) t_{A A *, A A *}\right],
\end{array}
$$

where matrix $T$ is three dimensional :

$$
T=\left(\begin{array}{ccc}
t_{(A B)^{1}(A B)^{1}} & t_{(A B)^{1}(A A *)^{1}} & -t_{(A B)^{1}(A * B)^{1}} \\
t_{(A A *)^{1}(A B)^{1}} & t_{(A A *)^{1}(A A *)^{1}} & t_{(A A *)^{1}(B A *)^{1}} \\
-t_{(B A *)^{1}(B A)^{1}} & t_{(B A *)^{1}(A A *)^{1}} & t_{(B A *)^{1}(B A *)^{1}}
\end{array}\right),
$$


and determinant of $T$ is equal to :

$$
\operatorname{det}(T)=t_{(A B)^{1}(A B)^{1}}\left[t_{(A A *)^{1}(A A *)^{1}} t_{(B A *)^{1}(B A *)^{1}}-\left(t_{(B A *)^{1}(A A *)^{1}}\right)^{2}\right]
$$

Likewise $l_{A}^{(0)}$ (equation (33)), $l_{A *}^{(0)}$ is a function of the four jump frequencies of $A$ :

$$
\begin{aligned}
l_{A^{*}}^{(0)} \simeq c_{v} c_{A^{*}}\left[c_{A}^{18} \omega_{A v}^{0}\right. & +7 c_{B} c_{A}^{17} \omega_{A v}^{4}+4 c_{B} c_{A}^{17} y_{B v} \omega_{A v}^{1}+7 c_{B} c_{A}^{17} y_{B v} \omega_{A v}^{3} \\
& \simeq c_{v} c_{A^{*}} \omega_{A v}^{0}\left[1-c_{B}\left(18-14 \frac{\omega_{A v}^{4}}{\omega_{A v}^{0}}-4 \frac{\omega_{A v}^{1}}{\omega_{A v}^{0}} \frac{\omega_{A v}^{4}}{\omega_{A v}^{3}}\right)\right] .
\end{aligned}
$$

Following the same scheme, terms entering equation (45) are calculated to first order in $c_{B}$ :

$$
\begin{gathered}
t_{(A A *)^{1},(A A *)^{1}} \simeq \omega_{A v}^{0} c_{A} c_{A *} c_{v}\left[11-c_{B}\left(17 * 11-142 \frac{\omega_{A v}^{4}}{\omega_{A v}^{0}}-52 \frac{\omega_{A v}^{1}}{\omega_{A v}^{0}} \frac{\omega_{A v}^{4}}{\omega_{A v}^{3}}\right)\right] \\
t_{(B A *)^{1},(A A *)^{1}} \simeq-\omega_{A v}^{0} c_{A *} c_{v} c_{B}\left(\frac{\omega_{A v}^{4}}{\omega_{A v}^{0}}+6 \frac{\omega_{A v}^{1}}{\omega_{A v}^{0}} \frac{\omega_{A v}^{4}}{\omega_{A v}^{3}}\right), \\
t_{(A * B)^{1},(A * \sigma)^{1}}(\sigma \equiv B)=t_{(A * B)^{1},(A * B)^{1}}^{\prime} c_{A *} c_{v} c_{B}^{2} \\
t_{(B A *)^{1},(B A *)^{1}} \simeq t_{(A * B)^{1},(A * B)^{1}}^{\prime} c_{A *} c_{v} c_{B}^{2}+c_{A *} c_{v} c_{B}\left(7 \omega_{A v}^{4}+2 \omega_{A v}^{1} \frac{\omega_{A v}^{4}}{\omega_{A v}^{3}}+9 \omega_{B v} \frac{\omega_{A v}^{4}}{\omega_{A v}^{3}}\right),
\end{gathered}
$$

and

$$
\begin{aligned}
m_{A *}(A, 1) \simeq & -\omega_{A v}^{0} c_{A} c_{A *} c_{v}\left[1-c_{B}\left(17-11 \frac{\omega_{A v}^{4}}{\omega_{A v}^{0}}-6 \frac{\omega_{A v}^{1}}{\omega_{A v}^{0}} \frac{\omega_{A v}^{4}}{\omega_{A v}^{3}}\right)\right. \\
& m_{A *}(B, 1) \simeq \omega_{A v}^{0} c_{A *} c_{v} c_{B}\left(-3 \frac{\omega_{A v}^{4}}{\omega_{A v}^{0}}+2 \frac{\omega_{A v}^{1}}{\omega_{A v}^{0}} \frac{\omega_{A v}^{4}}{\omega_{A v}^{3}}\right)
\end{aligned}
$$

We write down the resulting $L_{A^{*} A^{*}}$ :

$$
L_{A^{*} A^{*}} / \beta=l_{A^{*}}^{(0)}-\frac{N_{A^{*}}}{\operatorname{det}(T)}
$$


where the denominator is equal to :

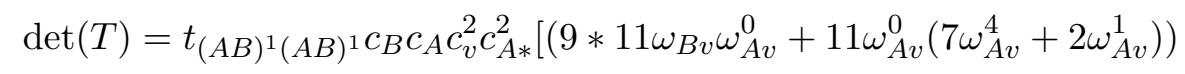

$$
\begin{aligned}
& +c_{B}\left(9 \omega_{B v}\left(52 \omega_{A v}^{1}+142 \omega_{A v}^{4}-187 \omega_{A v}^{0}\right)+11 \omega_{A v}^{0} t_{(A * B)^{1},(A * B)^{1}}^{\prime}-1309 \omega_{A v}^{0} \omega_{A v}^{4}\right. \\
& \left.\left.-374 \omega_{A v}^{0} \omega_{A v}^{1}+993\left(\omega_{A v}^{4}\right)^{2}+636 \omega_{A v}^{1} \omega_{A v}^{4}+68\left(\omega_{A v}^{1}\right)^{2}\right)\right]
\end{aligned}
$$

and the numerator is equal to:

$$
\begin{aligned}
& N_{A^{*}}=2 t_{(A B)^{1}(A B)^{1}} c_{A} c_{A *}^{3} c_{v}^{3} c_{B} \omega_{A v}^{0}\left\{\omega_{A v}^{0}\left(7 \omega_{A v}^{4}+2 \omega_{A v}^{1}+9 \omega_{B v}\right)\right. \\
& +c_{B}\left[\omega_{A v}^{0} t_{(A * B)^{1},(A * B)^{1}}^{\prime}-245 \omega_{A v}^{4} \omega_{A v}^{0}-70 \omega_{A v}^{1} \omega_{A v}^{0}+259\left(\omega_{A v}^{4}\right)^{2}\right. \\
& \left.\left.+44\left(\omega_{A v}^{1}\right)^{2}+28 \omega_{A v}^{1} \omega_{A v}^{4}+9 \omega_{B v}\left(+22 \omega_{A v}^{4}+12 \omega_{A v}^{1}-35 \omega_{A v}^{0}\right)\right]\right\}
\end{aligned}
$$

Finally, self-diffusion coefficient to first order in $c_{B}$ is defined through a zero order term $D_{A^{*}}\left(c_{B}=0\right)$ and an enhancement factor $b: D_{A^{*}}=D_{A^{*}}\left(c_{B}=0\right)\left(1+b c_{B}\right)$ with $b=b_{f}+b_{\omega} \cdot D_{A^{*}}\left(c_{B}=0\right)$ is equal to :

$$
D_{A^{*}}\left(c_{B}=0\right)=c_{v} \omega_{A v}^{0} \frac{9}{11}
$$

where $\frac{9}{11}$ is a first shell approximation of $f_{0}$. The contribution $b_{\omega}$ to the enhancement factor describes the variation of the mean frequency of $\mathrm{A}$ atoms due to the presence of solutes :

$$
b_{\omega}=-\left(18-14 \frac{\omega_{A v}^{4}}{\omega_{A v}^{0}}-4 \frac{\omega_{A v}^{1}}{\omega_{A v}^{0}} \frac{\omega_{A v}^{4}}{\omega_{A v}^{3}}\right)
$$

Treatment by Lidiard (1960) was assuming that enhancement coming from the correlations was negligible which is equivalent to put $f_{A}=f_{0}$ at any concentration. The resulting enhancement was then equal to $b_{\omega}$. 
While $b_{f}$ describes the enhancement of the correlation factor :

$$
\left.b_{f}=-2\left(1-\frac{9}{11}\right) \frac{\omega_{A v}^{4}}{\omega_{A v}^{0}} \frac{\frac{\omega_{B v}}{\omega_{A v}^{1}}\left(-27+18 \frac{\omega_{A v}^{1}}{\omega_{A v}^{3}}\right)+\frac{1}{9}\left(164 \frac{\omega_{A v}^{1}}{\omega_{A v}^{3}}+389 \frac{\omega_{A v}^{3}}{\omega_{A v}^{1}}-472\right)}{9 \frac{\omega_{B v}}{\omega_{A v}^{1}}+7 \frac{\omega_{A v}^{3}}{\omega_{A v}^{1}}+2}\right) .
$$

It is the first time that $b_{f}$ is given in an analytical form which allows to study its general behaviour with respect to each frequency ratio and to compare it with other analytical approaches available in some limiting cases.

\section{MONTE CARLO SIMULATIONS}

\section{A. The atomic diffusion model}

The probability per time unit of a single jump of an atom $\alpha$ at a site $i$ into a vacancy $v$ at a site $j$ has the "thermally activated" form

$$
\widehat{w}_{i j}^{\alpha v}(\mathbf{n})=\omega_{i j}^{\alpha v} \exp \left\{-\beta\left[\widehat{E}_{i j}^{\alpha v}(\mathbf{n})-\widehat{H}(\mathbf{n})\right]\right\}
$$

where we assume that the attempt frequency $\omega_{i j}^{\alpha v}$ is independent of the configuration ; the activation barrier is the difference between the energy of the system, $\widehat{E}^{(s)}{ }_{i j}^{\alpha v}$, with atom $\alpha$ at the saddle point position between sites $i$ and $j$ and the energy of the initial configuration $\mathbf{n}, \widehat{H}(\mathbf{n})$. Interactions which are not modified during the jump process do not contribute to the activation barrier, hence the activation barrier only depends on the local environment of sites $i$ and $j$. Interactions are assumed to be limited to the first nearest neighbour interactions both at the substitutional position $\left(V_{a b}\right)$ and at the saddle point positions $\left(V_{a b}^{s}\right)$. As a result the single jump probability (58) takes the final form

$$
\widehat{w}_{i j}^{\alpha v}(\mathbf{n})=\nu_{\alpha} \exp \left(-\beta\left[\sum_{k, \beta} \gamma_{\left(i^{\prime} k\right)^{1}}^{\prime} V_{\alpha \beta}^{s}-\left(\sum_{\mathbf{k} \neq \mathbf{j}, \beta} \gamma_{(i k)^{1}} V_{\alpha \beta}+\sum_{k, \beta} \gamma_{(j k)^{1}} V_{v \beta}\right)\right]\right.
$$


where $\nu_{\alpha}$ is the attempt jump frequency, the first term in the exponential corresponds to the new binding energies created at the saddle point and the second term between parenthesis to the binding energies to be cut by the exchanging species at initial state. In presence of one atom $B$ in the simulation box, the are only five jump frequencies to be distinguished. And the dependence upon the microscopic parameters of the three frequency ratios controlling the enhancement factor $b$ are known :

The corresponding jump frequency ratios are equal to :

$$
\begin{array}{r}
\frac{\omega_{A v}^{4}}{\omega_{A v}^{0}}=\exp \beta\left(V_{A B}-V_{A A}\right), \\
\frac{\omega_{A v}^{3}}{\omega_{A v}^{1}}=\exp \beta\left(V_{A B}^{s}-V_{A A}^{s}+V_{A A}-V_{A B}\right) \\
\frac{\omega_{B v}}{\omega_{A v}^{1}}=\frac{\nu_{B}}{\nu_{A}} \exp \beta\left(-4 \mathbf{V}_{\mathbf{B A}}^{\mathbf{s}}+3 V_{A A}^{s}+V_{A B}^{s}+10 V_{A B}-10 V_{A A}\right) .
\end{array}
$$

Note that in reality there is more than one B atom in the simulation box and other jump frequencies may be required during the simulation. In opposition to Belova and Murch (2003b), we keep them and we affect to them the value provided by equation (59). Three sets of parameters are proposed to explore the behaviour of $b$ with respect to the variation of the three frequency ratios one by one.

First set of parameters corresponds to : $V_{A A}=-1.1984 \mathrm{eV}, V_{B B}=-1.2344 \mathrm{eV}, V_{A B}=-1.1984 \mathrm{eV}$, $V_{A v}=0, V_{B v}=-0.0016 \mathrm{eV}, V_{A A}^{s}=-2.4 \mathrm{eV}, V_{A B}^{s}=-2.4597, V_{B A}^{s}=V_{B B}^{s}=-2.9 \mathrm{eV}, \nu_{A}=5.10^{15}$ and and $\nu_{B}=8.30404 \times 10^{5} x$ so that $\omega_{A v}^{4} / \omega_{A v}^{0}=1, \omega_{A v}^{3} / \omega_{A v}^{1}=0.5$ and $\omega_{B v} / \omega_{A v}^{1}=x$.

Second set of parameters corresponds to : $V_{A A}=-1 \mathrm{eV}, V_{B B}=-1.4328 \mathrm{eV}, V_{A B}=-1.1984 \mathrm{eV}, V_{A v}=$ $0, V_{B v}=-0.2 \mathrm{eV}, V_{A A}^{s}=-2.4 \mathrm{eV}, V_{B A}^{s}=V_{B B}^{s}=-2.9 \mathrm{eV}, \nu_{A}=5.10^{15}$ and $V_{A B}^{s}=-2.5984+k_{B} T \ln (x)$ and $\nu_{B}=4.15867 \times 10^{15} / x$ so that $\omega_{A v}^{4} / \omega_{A v}^{0}=0.1, \omega_{A v}^{3} / \omega_{A v}^{1}=x$ and $\omega_{B v} / \omega_{A v}^{1}=0.1$. 
Third set of parameters corresponds to : $V_{A A}=-0.9-k_{B} T \ln x e V, V_{B B}=-0.936+k_{B} T \ln x e V$, $V_{A B}=-0.9 \mathrm{eV}, V_{A v}=V_{B v}=0, V_{A A}^{s}=-2.4 \mathrm{eV}, V_{B A}^{s}=V_{B B}^{s}=0 \mathrm{eV}, V_{A A}^{s}=-2.75 \times k_{B} T \ln (x)$, $V_{A B}^{s}=-1.75 \times k_{B} T \ln (x), \nu_{A}=\nu_{B}=10^{55}$ so that $\omega_{A v}^{4} / \omega_{A v}^{0}=x, \omega_{A v}^{3} / \omega_{A v}^{1}=1$ and $\omega_{B v} / \omega_{A v}^{1}=1$.

Note that the three sets of parameters are adjusted in such a way that there is no trapping of vacancies on solutes which would have modified the concentration of vacancy in the regions of pure A. The affinity between $A$ and $B$ is fixed by the ratio:

$$
\frac{\omega_{A v}^{4}}{\omega_{A v}^{3}}=\frac{\omega_{A v}^{4}}{\omega_{A v}^{0}} \exp \left[\beta\left(V_{A v}-V_{B v}\right)\right]
$$

which is very close to one for the three parameter sets so that the concentration of vacancy in the matrix is not modified by the addition of solutes $B$. In real materials, vacancies interact with solutes but sources and sinks like grain-boundaries, interfaces or dislocations are usually supposed to be efficient enough to guarantee a vacancy concentration in the matrix very close to the value measured in pure metal.

\section{B. Conditions of simulation}

The simulation box contains $16^{3}=4096$ sites among which 45 sites are occupied by $B$ atom and one by a vacancy. The concentration in solute atoms is then equal to $c_{B}=0.0109$ which at the temperature of the simulations $(T=1000 K)$ is checked to be inferior to the solubility limit, $c_{0}$ :

$$
c_{0}=\exp \left\{W / k_{B} T\right\}=0.081,
$$

where $W=12 / 2\left(V_{A A}+V_{B B}-2 V_{A B}\right)=-0.216 \mathrm{eV}$ is a negative ordering energy which simulates a system with clustering tendencies. Note that MC simulations were performed with lower values of 
$c_{B}$ to check that the slope of the self-diffusion coefficient with respect to $c_{B}$ remains constant in this concentration range. Each MC value is an average of 10000 (or 50000 if necessary) measures. Each measure of diffusion coefficient is a result of a Monte carlo run of $10^{7}$ steps. The calculated standard error of the mean (SEM) (the mean square divided by the square root of the number of measures) does not exceed $0.1 \%$ of the diffusion coefficient value. $b$ is obtained as the difference between the diffusion coefficient measured at $c_{B}=0.0109$ minus the same diffusion coefficient measured in the pure metal A divided by $c_{B}=0.0109$. Error on $b$ does not exceed 0.1 .

\section{DISCUSSION}

While the $L_{i j}$ of a binary alloy have been precisely known since 1981 (Allnatt 1981), a reliable model for the enhancement factor $b$ is still lacking. From the previous sections, we obtained a new analytical formulae of $b$ which is compared to the MC simulations and the available models. Some of the differences with the MC simulations are explained in terms of the vacancy paths in relation with the range of the effective interactions considered.

\section{A. The fcc random alloy}

First historical test of the multi-frequency model was to reduce the alloy to a random lattice gas which is equivalent to say that the influence of solutes on the jump rate of the solvent is negligible and that there is only one solvent jump frequency : $\omega_{A v}=\omega_{A v}^{0}=\omega_{A v}^{1}=\omega_{A v}^{3}=\omega_{A v}^{4}$. This limiting case appeared to be a crucial test of the multi-frequency models (Allnatt and Lidiard 1987). Moreover, recent Monte Carlo simulations (Belova and Murch 2003b) established how wrong were the models. It appeared 
that the so-called random lattice gas models starting with an ideal solid solution and associating to each chemical species a unique jump rate were in better agreement with the Monte Carlo simulations. Among the various random alloy model, Belova and Murch (2003b) have shown that predictions of the correlation factors of Moleko, Allnatt and Allnatt (1989) were almost equal to the exact Monte Carlo simulations of a random alloy even at very low concentration of solutes which is not the case of the random alloy model of Manning (1971) . In the following, the formulae of Moleko, Allnatt and Allnatt (1989) will be stated as the reference :

$$
b(\mathbf{M} . \mathbf{A} . \mathbf{A} .)=\frac{2\left(1-f_{0}\right)\left(\omega_{B v}-\omega_{A v}\right)}{\omega_{B v}+\omega_{A v}+\left(1-f_{0}\right)\left(\omega_{A v}-\omega_{B v}\right)},
$$

where the vacancy concentration is assumed to be very low (see Belova and Murch 2003b to obtain the details of the derivation).

The SCMF model (equations (56) (57)) is simplified by assuming that every solvent jump frequency is equal to $\omega_{A v}$ :

$$
b=2\left(1-\frac{9}{11}\right) \frac{\left(\omega_{B v}-\omega_{A v}\right)}{\omega_{B v}+\omega_{A v}} .
$$

A previous publication, based on the same atomic diffusion model and using the same self-consistent mean field formalism, yielded more general results since it considered the whole series of effective pair interactions at infinite range and any isotropic crystallographic structure (fcc, body centered cubic, diamond, etc.). The principle was to neglecting variations of jump frequencies upon the configuration of nearby atoms and to assume that jump frequencies appearing in equation (28) were all equal which was coherent with the Bragg Williams statistical approximation used to calculate the thermodynamic averages. The resulting $L_{i j}$ depended on two effective jump frequencies, $\omega_{A v}$ and $\omega_{B v}$, the alloy 
composition and the correlation factor for self-diffusion, $f_{0}$. The corresponding expression of $b$ coincides with equation (64) unless the constant $\left(1-\frac{9}{11}\right)$ is replaced by the more general quantity $\left(1-f_{0}\right)$. On the other hand a discussion given in (Nastar et al. 2000) predicts that equation (64) is quite satisfying as long as the exchange frequencies are not too different. Indeed the effective Hamiltonian is truncated after pair interactions and self-consistency is satisfied until two-point averages only. Time derivatives of N-point averages $(N>2)$ are proportional to $\left(\omega_{A}-\omega_{B}\right)$. The larger the difference between effective frequencies the worse the self-consistency of kinetic equations. Indeed, it is observed that the difference between equations $(63)$ and $(64)$ increases with $\left(\omega_{A}-\omega_{B}\right)$. In summary, we expect that a critical test of the present model will be the behaviour of $b$ for high values of $\left(\omega_{B v}-\omega_{A v}\right)$. For example, when $\omega_{B v}$ (resp. $\omega_{A v}$ ) is taken to be zero, $b$ (M.A.A.) is found to be equal to -0.559 (resp. 0.559) while the present $b$ is equal to -0.437 (resp. 0.437).

[Insert table 1 about here]

However, table (I) shows that a relative error of around $20 \%$ on the value of $b$ is not so critical compared to the errors made by the multi-frequency models which in some cases do not predict the right sign even. The enhancement factor should vanish when jump frequency of solute is equal to the solvent jump frequency which is verified by all the random alloy model and the present model but not by the five frequency models. It is even more surprising that an improved calculation (Ishioka and Koiwa 1984) which considers larger radius for the estimation of the partial correlation factors appears to be worse than the initial approximation of H. M. (1967). Note that the predictions of Lidiard 
(1960) are not given in table (I) since it would give 0 at any value of the jump rates. As a conclusion already noted down by Allnatt and Lidiard (1987) and recently confirmed by Monte Carlo simulations of Belova and Murch (2003), the multi-frequency models are inaccurate when they are used to predict the effect of solute on self-diffusion in a random alloy.

\section{B. The fcc interacting alloy}

In opposition to the last five-frequency models (Howard and Manning 1967, Ishioka and Koiwa 1984), the present model provides with an analytical expression of $b$ (equations (56) and (57)). As shown by Howard and Manning (1967), the enhancement factor depends on three ratios, $\omega_{A v}^{3} / \omega_{A v}^{1}, \omega_{B v} / \omega_{A v}^{1}$ and $\omega_{A v}^{4} / \omega_{A v}^{0}$. The two first ratios represent the relative probabilities of the possible jumps a vacancy can make when it is at a $\mathrm{nn}$ site of a solute atom. And the last ratio, $\omega_{A v}^{4} / \omega_{A v}^{0}$ represents the relative probability of the jumps a vacancy can make when it is at a next nn site of a solute atom. The ratio $\omega_{A v}^{4} / \omega_{A v}^{3}$ which determines the probability of forming a pair $B v$ is not involved as long as sinks and sources of the system manage to maintain the vacancy concentration in the matrix at the corresponding value in pure $A$. We shall limit the discussion to the comparison between the present model and the previous five frequency models. No comparison is possible with other mean field approaches such as the Path Probability Method (Sato and Kikuchi 1983) and the Taylor series expansion of the time correlation functions (Qin et al. 1998) or more empirical techniques (Stolwijk 1981, Bakker 1979) since no one makes appear the five jump frequencies in the dilute limit.

Although, the Lidiard model (1960) is limited to the description of the effect of solute on the mean solvent jump frequency $\left(b_{\omega}\right)$, it is represented in the figures so that the correlation effect contribution, 
$b_{f}$, can be directly measured by making the difference with the values of Lidiard. Note that the MC simulations and every theory agree on the estimation of $b_{\omega}$, the discrepancies with the MC results are due to an approximate estimation of the correlation effects.

The jump frequency of solutes can have a significant effect on the self-diffusion by modifying its correlation factor. This effect is omitted by the first model of Lidiard (1960) which is limited to $b=b_{\omega}$ (equation (56)). The SCMF model predicts an increasing of $b$ with $\omega_{B v} / \omega_{A v}^{1}$ no matter the value of $\omega_{A v}^{3} / \omega_{A v}^{1}$ and $\omega_{A v}^{4} / \omega_{A v}^{0}$. Indeed

$$
\frac{\partial b}{\partial\left(\frac{\omega_{B v}}{\omega_{A v}^{1}}\right)}=4\left(1-\frac{9}{11}\right)\left(\frac{\omega_{A v}^{4}}{\omega_{A v}^{0}}\right)\left(\frac{\omega_{A v}^{3}}{\omega_{A v}^{1}}\right) \frac{\left(8 \frac{\omega_{A v}^{1}}{\omega_{A v}^{3}}-17\right)^{2}}{\left(9 \frac{\omega_{B v}}{\omega_{A v}^{1}}+7 \frac{\omega_{A v}^{3}}{\omega_{A v}^{1}}+2\right)^{2}}
$$

is always positive. This tendency already predicted by H.M. (Howard and Manning 1969) is in agreement with the Monte Carlo simulations (figures (1) and (2)). We choose to represent MC simulations of Belova and Murch (2003a) in figure (2) to explore a new set of jump frequencies but also to make sure that a positive variation of $b$ with $\omega_{B v} / \omega_{A v}^{1}$ is verified by different authors. The SCMF theory predicts a very small variation of $b$ in agreement with the MC simulations while it underestimates the slowing down of self-diffusion due to correlation effects. Calculation of I.K. (Ishioka and Koiwa 1984) was proved to be not accurate in the random alloy limit. Figures (1) and (2) reveal that it is still not accurate when it is examined in the region where the various host atom jump frequencies are close to one another. Moreover, the prediction of a negative slope of $b$ with respect to $\omega_{B v} / \omega_{A v}^{1}$ extends beyond the set of frequencies represented in figures (1) and (2)) : at $\omega_{A v}^{4} / \omega_{A v}^{0}=0.1$ and $\omega_{A v}^{3} / \omega_{A v}^{1}>0.1$; and also at $\omega_{A v}^{4} / \omega_{A v}^{0}=1$ and $0.25<\omega_{A v}^{3} / \omega_{A v}^{1}<5$. For some values of the jump frequencies, the curves of I.K. and H.M. are above the curve associated to the Lidiard theory (1960) and predict then a speed up of the self-diffusion coefficient by the correlation effects, which is in contradiction with the MC 
simulations.

[Insert figure 1 and 2 about here]

The fall of $b$ at low values of $\omega_{A v}^{3} / \omega_{A v}^{1}$ is a general feature occurring at any value of the other two ratios (figure (3) and Belova and Murch 2003a).

[Insert figure 3 about here]

A schematic representation given in figure (4) helps to understand the role of correlations in relation with the range of the effective interactions. It represents the possible jumps of a vacancy after a first jump with a tracer in a fcc lattice. Two initial jumps are represented, $\omega_{1}$ (case a)) and $\omega_{0}$ (case b)). In both cases, low values of $\omega_{A v}^{3} / \omega_{A v}^{1}$ enhance the jump back of the vacancy in opposition to the escaping jumps which lead to a slowing down of the self-diffusion by the correlation effects. The role of the nn effective interactions appears clearly in equation (9). They require to determine what are the chemical species on the $\mathrm{nn}$ sites of the migrating atom (tracer). In figure (4), the solute atom of case a) is seen by the effective interactions since it is nn of the tracer but not the one of case b). We understand then that some of the correlation effects are missed by the SCMF when the effective hamiltonian is restricted to nn effective interactions. Ishioka and Koiwa (1984) which consider longer paths of the 
vacancy and more details of the local environment around the vacancy is more efficient to describe such a strong correlation effect.

[Insert figure 4 about here]

The MC simulations of figure (5) reveal a non-monotonous variation of $b_{f}$ with respect to $\omega_{A v}^{4} / \omega_{A v}^{0}$. Indeed at values of $\omega_{A v}^{4} / \omega_{A v}^{0}$ above 5, correlations effects enhance the self-diffusion. Note that this phenomenon was not observed by Belova and Murch (2003a) perhaps because self-diffusion was not studied for values of $\omega_{A v}^{4} / \omega_{A v}^{0}$ beyond 5. H. M. and I. K. overestimate the slowing down due to correlation effects and do not predict the reverse trend at higher values of the ratio $\omega_{A v}^{4} / \omega_{A v}^{0}$, while the present theory predicts a $b_{f}$ equal to zero which appears to be a better approximation of $b_{f}$. Although the present model moves away from the MC simulations at higher values of $\omega_{A v}^{4} / \omega_{A v}^{0}$. An acceleration of self-diffusion by the correlation effects means that the solvent correlation factor is higher than $f_{0}$ but still below 1 .

[Insert figure 5 about here]

A schematic diagram drawn in figure (6) represents the possible paths of a vacancy after a $\omega_{3}$ or a $\omega_{0}$ jump type. Note that in the case of an alloy simulated by the third parameter set (defined in section 
IV), initial jumps of type $\omega_{1}$ and $\omega_{4}$ would show no preferential path of a vacancy. Figure (6) shows that in both cases a high value of $\omega_{4} / \omega_{0}$ enhances the escape frequency of a vacancy compared to a coming back. It is natural then to observe an acceleration of the self-diffusion by the correlation effects. Note that the nn effective interactions do not see the solute atom so that the vacancy follows a random path after the initial jump which explains that the SCMF curve meets the Lidiard curve in figure (5).

[Insert figure 6 about here]

The model has been successfully tested in the region of the random alloy (figures (1) and (2)). In an interacting alloy, the SCMF results are observed to be in reasonable agreement with the MC simulations and well reproduce the main tendencies : large slowing down of the self-diffusion due to correlations at low ratio $\omega_{3} / \omega_{1}$ and weak correlation effects in an alloy associated to the third parameter set (figure 5). Moreover, the limitations of the model are easily connected to the range of the effective interactions considered. A significant improvement of the model would be obtained if the effective Hamiltonian was extended to the next nearest neighbour effective interactions.

\section{CONCLUSION}

SCMF kinetic equations based on an atomic model of atom-vacancy exchange frequencies are written in the general case of a multi-component alloy with any crystallographic structure. They lead to an 
expression of the phenomenological coefficients as a function of time dependent effective interactions which are solved using the the kinetic equations of the next moments of the distribution function under steady state conditions. An explicit calculation is carried out for the case of a fcc matrix of $A$ atoms containing a small amount of $B$ atoms and isotope atoms $\left(A^{*}\right)$. The phenomenological coefficients, $L_{A A}, L_{A B}$ and $L_{B B}$, are equivalent to the first shell formulae of the five-frequency model (Howard and Lidiard 1963, 1964). A new analytical model for evaluating the effect of solute atoms on the selfdiffusion coefficient is given. In the particular case of a random alloy, the present SCMF approximation is found to be equivalent to a previous formulation based on a lower statistical approximation but valid at any concentration of a multi-component alloy. In agreement with the Monte Carlo simulations the self-diffusion coefficient is increasing with the solute jump frequency in opposition to the previous five-frequency model (Ishioka and Koiwa 1984). Although a good quantitative agreement is obtained (within $20 \%$ ), the discrepancy is recognized to be due to the fact that pair effective interactions are considered only with a neglect of the triplet interactions. In an alloy with interactions, the SCMF prediction of the self-diffusion coefficient reproduces the main tendencies of the Monte Carlo simulations based on the same atomic model, with in general, a better agreement than the previous five-frequency models. When the quantitative agreement is not satisfying (for example at low ratio of $\left.\omega_{3} / \omega_{1}\right)$, it can be explained by the fact that nearest neighbour effective interactions are considered only which greatly reduces the level of description of the diffusion paths offered to a vacancy after a first exchange. As a consequence the main discrepancies can be easily corrected by an extension of the effective Hamiltonian to interactions with a larger range. This approach is promising since it reconciles a diffusion model devoted to concentrated alloys with a multi-frequency approach usually restricted 
to dilute alloys. The objective will be to give a multi-frequency formulation of the phenomenological coefficients of a concentrated alloy. It will allow to predict negative values of the non-diagonal transport coefficients which is not possible with the actual diffusion models of concentrated alloys.

\section{APPENDIX A}

Following equation (3), the distribution function is divided into two terms :

$$
\begin{aligned}
\frac{d\left\langle n_{i}^{\alpha} n_{j}^{\beta} n_{k}^{\gamma} \ldots\right\rangle}{d t}= & \sum_{s \neq i \neq j \neq \ldots}\left\langle n_{j}^{\beta} n_{k}^{\gamma} \ldots\left[n_{s}^{\alpha} n_{i}^{v} \widehat{\omega}_{s i}^{\alpha v}-n_{i}^{\alpha} n_{s}^{v} \widehat{\omega}_{i s}^{\alpha v}\right] \widehat{P}_{1}(\mathbf{n}, t)\right\rangle^{(0)}+ \\
& \sum_{s \neq i \neq j \neq \ldots}\left\langle n_{i}^{\alpha} n_{k}^{\gamma} \ldots\left[n_{s}^{\beta} n_{j}^{v} \widehat{\omega}_{s j}^{\beta v}-n_{j}^{\beta} n_{s}^{v} \widehat{\omega}_{j s}^{\beta v}\right] \widehat{P}_{1}(\mathbf{n}, t)\right\rangle^{(0)}+\ldots,
\end{aligned}
$$

where $\langle\ldots\rangle^{(0)}$ means averaging over the equilibrium distribution function $\widehat{P}_{0}(\mathbf{n})$. Expansion of $\widehat{P}_{1}(\mathbf{n})$ to first order in $\widehat{h}(t)$ and $\delta \mu(t)$ gives :

$$
\begin{aligned}
\frac{d\left\langle n_{i}^{\alpha} n_{j}^{\beta} n_{k}^{\gamma} \ldots\right\rangle}{d t}= & \sum_{s \neq i \neq j \neq \ldots}\left\langle n_{j}^{\beta} n_{k}^{\gamma} \ldots\left[n_{s}^{\alpha} n_{i}^{v} \widehat{\omega}_{s i}^{\alpha v}-n_{i}^{\alpha} n_{s}^{v} \widehat{\omega}_{i s}^{\alpha v}\right]\left(1+\beta\left(\delta \Omega(t)+\sum_{\alpha, i} \delta \mu_{i}^{\alpha}(t) n_{i}^{\alpha}-\widehat{h}(t)\right)\right)\right\rangle^{(0)}+ \\
& \sum_{s \neq i \neq j \neq \ldots}\left\langle n_{i}^{\alpha} n_{k}^{\gamma} \ldots\left[n_{s}^{\beta} n_{j}^{v} \widehat{\omega}_{s j}^{\beta v}-n_{j}^{\beta} n_{s}^{v} \widehat{\omega}_{j s}^{\beta v}\right]\left(1+\beta\left(\delta \Omega(t)+\sum_{\alpha, i} \delta \mu_{i}^{\alpha}(t) n_{i}^{\alpha}-\widehat{h}(t)\right)\right)\right\rangle^{(0)}+\ldots(67)
\end{aligned}
$$

By construction of the exchange frequencies, detailed balance principle is satisfied at equilibrium :

$$
\widehat{P}_{0}(\mathbf{n}) n_{s}^{\alpha} n_{i}^{v} \widehat{\omega}_{s i}^{\alpha v}(\mathbf{n})=\widehat{P}_{0}\left(\mathbf{n}^{\prime}\right) n_{i}^{\prime \alpha} n_{s}^{\prime v} \widehat{\omega}_{i s}^{\alpha v}\left(\mathbf{n}^{\prime}\right)
$$

where configurations $\mathbf{n}$ and $\mathbf{n}^{\prime}$ differ by an exchange of atom $\alpha$ on site $i$ and a vacancy $v$ on a neighbouring site $s$. Equation (68) summed over all the configurations leads to the equality :

$$
\left\langle n_{s}^{\alpha} n_{i}^{v} \widehat{\omega}_{s i}^{\alpha v}\right\rangle^{(0)}=\left\langle n_{i}^{\alpha} n_{s}^{v} \widehat{\omega}_{i s}^{\alpha v}(\mathbf{n})\right\rangle^{(0)} .
$$


Therefore to first order in $\widehat{h}(t)$ and $\delta \mu(t)$, equation (67) simplifies to :

$$
\begin{array}{r}
\frac{d\left\langle n_{i}^{\alpha} n_{j}^{\beta} \ldots\right\rangle}{d t}=\beta \sum_{s \neq i \neq j \neq \ldots}\left\langle n_{j}^{\beta} \ldots\left[n_{s}^{\alpha} n_{i}^{v} \widehat{\omega}_{s i}^{\alpha v}\left(\delta \mu_{s}^{\alpha}-\delta \mu_{i}^{\alpha}-\widehat{h}_{s}^{\alpha}+\widehat{h}_{i}^{\alpha}\right)\right\rangle^{(0)}+\right. \\
\beta \sum_{\substack{s \neq i \neq j \neq \ldots \\
s \neq i}}\left\langle n_{i}^{\alpha} \ldots n_{i}^{\alpha} n_{s}^{\beta} n_{j}^{v} \widehat{\omega}_{s j}^{\beta v}\left(\delta \mu_{s}^{\beta}-\delta \mu_{j}^{\beta}-\widehat{h}_{s}^{\beta}+\widehat{h}_{j}^{\beta}\right)\right\rangle^{(0)}+\ldots
\end{array}
$$

Note that $\delta \Omega(t)$ disappears in the difference.

\section{APPENDIX B}

The calculation consists in dividing the sums into blocks of equivalent four-point averages and by using the antisymmetry property of the effective interactions summing each block in terms of the positive first nearest neighbour effective interaction. The estimation of each sum is listed in table (II), each cell corresponding to a summation over $p$ indices at fixed site $s$. To get the final result it suffices to sum the equivalent four-point averages over $s$. For example first term of the rhs of equation (25) results from the sum of the first three cells of the first column, each cell being weighted by the number of equivalent $s$ sites : $\left[(-4) * 1+(-2) * 2+(0) * 4 v_{(\alpha \sigma)^{1}}\right]\left(v_{(\alpha \sigma)^{1}} / v_{(\alpha \sigma)^{1}}\right)$.

\section{[Insert table 2 about here]}

\section{ACKNOWLEDGMENTS}

The authors are grateful to V. Barbe, P. Bellon, J. L. Bocquet, E. Clouet, B. Legrand, G. Martin and F.Soisson, for their judicious comments and their assistance. 


\section{REFERENCES}

[1] Allnatt, A. R., 1981, J. Phys. C : Solid State Phys., 14, 5453.

[2] Allnatt, A. R., and Lidiard, A. B., 1987, Acta metall., 35, 1555.

[3] Allnatt, A. R., and Lidiard, A. B., 1993, Atomic Transport in Solids (Cambridge : University Press).

[4] Bakker, H., 1979, Phil. Mag. A, 40, 525.

[5] Belova, I. V., and Murch, G. E., 2003a, Phil. Mag., 83, 377.

[6] Belova, I. V., and Murch, G. E., 2003b, Phil. Mag., 83, 393.

[7] Ducastelle, F., 1991, Order and Phase Stability in Alloys (Amsterdam : North-Holland).

[8] Howard, R. E., and Lidiard, A. B., 1963, J. Phys. Soc. Japan, 18(Suppl. 2), 197.

[9] Howard, R. E., and Lidiard, A. B., 1964, Rep. Prog. Phys., 27, 161.

[10] Howard, R. E., and Manning, J. R., 1967, Phys. Rev., 154, 561.

[11] Ishioka, S., and Koiwa, M., Phil. Mag. A, 1984, 50, 505.

[12] Kikuchi, R., and Sato, H., 1969, J. Chem. Phys., 51, 161.

[13] Lidiard A. B., 1960, Phil. Mag., 5, 1171.

[14] Manning, J. R., 1971, Phys. Rev. B, 1971, 4, 1111.

[15] Moleko, L. K., Allnatt, A. R., and Allnatt, E. L., 1989, Phil. Mag. A, 59, 141.

[16] Nastar, M., Dobretsov, V. Yu., and Martin, G., 2000, Phil. Mag. A, 80, 155.

[17] Qin, Z., Allnat, A. R., and Allnat, E. L., 1998, J. Phys. : Condens. Matter, 10, 5295.

[18] Sanchez, J. M., Ducastelle, F., and Gratias, D., Physica A, 1984, 128, 334.

[19] Sato, H., and Kikuchi, R., 1983, Phys. Rev. B, 28, 648.

[20] Sato, H., Ishikawa, T., and Kikuchi, R., 1985, J. Phys. Chem. Solids, 46, 1361. 
[21] N. A. Stolwijk, 1981, Phys. Stat. sol. (b), 105, 223.

[22] I. A. Szabo and D. L. Beke, 2004, Phys. Chem. Chem. Phys., 6, 3625.

[23] Vaks, V. G., 1996, Pis'ma Zh.eksp. teor. Fiz., 1996, 63, 447. Engl. Transl. JETP Lett., 1996, 63, 471. 
1

2

3

4

5

6

7

8

9

10

11

12

13

14

15

16

17

18

19

20

21

22

23

24

25

26

27

28

29

30

31

32

33

34

35

36

37

38

39

40

41

42

43

44

45

46

47

48

49

50

51

52

53

54

55

56

57

58

59

60

\begin{tabular}{|c|c|c|c|c|}
\hline & H.M. (1967) & I.K. (1983) & S.C.M.F. & M.A.A. (1989) \\
\hline$\omega_{B v}=0$ & 0.010 & 0.855 & -0.437 & -0.559 \\
$\omega_{B v}=\omega_{A v}$ & 0.215 & 0.163 & 0 & 0 \\
$\omega_{B v} / \omega_{A v}=10^{3}$ & 0.898 & -0.367 & 0.437 & 0.559 \\
\hline
\end{tabular}

TABLE I: The enhancement factor $b$ for different values of the ratio of jump frequencies associated to $A$ and $B$ in a binary alloy $A B$ without interaction predicted by the five-frequency models of H.M. (Howard and Manning 1967) and I.K. (Ishioka and Koiwa 1984) ; by the present model (SCMF) and by M.A.A. (Moleko, Allnatt and Allnatt 1989). 


\begin{tabular}{|c|c|c|c|c|}
\hline & $\begin{array}{c}\gamma_{(i j)^{1}} \sum_{p} \gamma_{(i s)^{1}} \\
\left(1-\gamma_{(s p)^{1}}\right)\left(1-\gamma_{(j p)^{1}}\right) \\
\left(1-\gamma_{(j s)^{1}}\right) \gamma_{(i p)^{1}} v_{i p}^{\alpha \sigma}\end{array}$ & $\begin{array}{c}\gamma_{(i j)^{1}} \sum_{p} \gamma_{(i s)^{1}} \\
\left(1-\gamma_{(s p)^{1}}\right) \gamma_{(j p)^{1}} \\
\left(1-\gamma_{(j s)^{1}}\right) \gamma_{(i p)^{1}} v_{i p}^{\alpha \sigma}\end{array}$ & $\begin{array}{c}\gamma_{(i j)^{1}} \sum_{p} \gamma_{(i s)^{1}} \\
\gamma_{(s p)^{1}}\left(1-\gamma_{(j p)^{1}}\right) \\
\left(1-\gamma_{(j s)^{1}}\right) \gamma_{(i p)^{1}} v_{i p}^{\alpha \sigma}\end{array}$ & $\begin{array}{c}\gamma_{(i j)^{1}} \sum_{p} \gamma_{(i s)^{1}} \\
\gamma_{(s p)^{1}} \gamma_{(j p)^{1}} \\
\left(1-\gamma_{(j s)^{1}}\right) \gamma_{(i p)^{1}} v_{i p}^{\alpha \sigma}\end{array}$ \\
\hline $1 s \in\left(i^{+}\right)$ & $-4 v_{(\alpha \sigma)^{1}}$ & 0 & 0 & $2 v_{(\alpha \sigma)^{1}}$ \\
\hline $2 s \in(i)$ & $-2 v_{(\alpha \sigma)^{1}}$ & $v_{(\alpha \sigma)^{1}}$ & $v_{(\alpha \sigma)^{1}}-2 v_{(\alpha \sigma)^{1}}$ & $v_{(\alpha \sigma)^{1}}$ \\
\hline \multirow[t]{2}{*}{$4 s \in\left(i^{-}\right)$} & $v_{(\alpha \sigma)^{1}}-v_{(\alpha \sigma)^{1}}$ & $2 v_{(\alpha \sigma)^{1}}$ & $-2 v_{(\alpha \sigma)^{1}}$ & 0 \\
\hline & $\begin{array}{c}\gamma_{(i j)^{1}} \sum_{p} \gamma_{(i s)^{1}} \\
\left(1-\gamma_{(s p)^{1}}\right)\left(1-\gamma_{(j p)^{1}}\right) \\
\gamma_{(j s)^{1}} \gamma_{(i p)^{1}} v_{i p}^{\alpha \sigma}\end{array}$ & $\begin{array}{c}\gamma_{(i j)^{1}} \sum_{p} \gamma_{(i s)^{1}} \\
\left(1-\gamma_{(s p)^{1}}\right) \gamma_{(j p)^{1}} \\
\gamma_{(j s)^{1}} \gamma_{(i p)^{1}} v_{i p}^{\alpha \sigma}\end{array}$ & $\begin{array}{c}\gamma_{(i j)^{1}} \sum_{p} \gamma_{(i s)^{1}} \\
\gamma_{(s p)^{1}}\left(1-\gamma_{(j p)^{1}}\right) \\
\gamma_{(j s)^{1}} \gamma_{(i p)^{1}} v_{i p}^{\alpha \sigma}\end{array}$ & $\begin{array}{c}\gamma_{(i j)^{1}} \sum_{p} \gamma_{(i s)^{1}} \\
\gamma_{(s p)^{1}} \gamma_{(j p)^{1}} \\
\gamma_{(j s)^{1}} \gamma_{(i p)^{1}} v_{i p}^{\alpha \sigma}\end{array}$ \\
\hline $2 s \in\left(i^{+}\right)$ & $-4 v_{(\alpha \sigma)^{1}}$ & $v_{(\alpha \sigma)^{1}}$ & $v_{(\alpha \sigma)^{1}}$ & 0 \\
\hline \multirow[t]{2}{*}{$2 s \in(i)$} & $v_{(\alpha \sigma)^{1}}-2 v_{(\alpha \sigma)^{1}}$ & $v_{(\alpha \sigma)^{1}}$ & $-2 v_{(\alpha \sigma)^{1}}$ & $v_{(\alpha \sigma)^{1}}$ \\
\hline & $\begin{array}{c}\gamma_{(i j)^{1}} \sum_{p} \gamma_{(i s)^{1}} \\
\left(1-\gamma_{(i p)^{1}}\right)\left(1-\gamma_{(j p)^{1}}\right) \\
\left(1-\gamma_{(j s)^{1}}\right) \gamma_{(s p)^{1}} v_{s p}^{\alpha \sigma}\end{array}$ & $\begin{array}{c}\gamma_{(i j)^{1}} \sum_{p} \gamma_{(i s)^{1}} \\
\left(1-\gamma_{(i p)^{1}}\right) \gamma_{(j p)^{1}} \\
\left(1-\gamma_{(j s)^{1}}\right) \gamma_{(s p)^{1}} v_{s p}^{\alpha \sigma}\end{array}$ & $\begin{array}{c}\gamma_{(i j)^{1}} \sum_{p} \gamma_{(i s)^{1}} \\
\gamma_{(i p)^{1}}\left(1-\gamma_{(j p)^{1}}\right) \\
\left(1-\gamma_{(j s)^{1}}\right) \gamma_{(s p)^{1}} v_{s p}^{\alpha \sigma}\end{array}$ & $\begin{array}{c}\gamma_{(i j)^{1}} \sum_{p} \gamma_{(i s)^{1}} \\
\gamma_{(i p)^{1}} \gamma_{(j p)^{1}} \\
\left(1-\gamma_{(j s)^{1}}\right) \gamma_{(s p)^{1}} v_{s p}^{\alpha \sigma}\end{array}$ \\
\hline $1 s \in\left(i^{+}\right)$ & $3 v_{(\alpha \sigma)^{1}}-v_{(\alpha \sigma)^{1}}$ & $v_{(\alpha \sigma)^{1}}$ & $-2 v_{(\alpha \sigma)^{1}}$ & 0 \\
\hline $2 s \in(i)$ & $2 v_{(\alpha \sigma)^{1}}-2 v_{(\alpha \sigma)^{1}}$ & 0 & $v_{(\alpha \sigma)^{1}}-2 v_{(\alpha \sigma)^{1}}$ & $v_{(\alpha \sigma)^{1}}$ \\
\hline $1 s \in\left(i^{-}\right)$ & $v_{(\alpha \sigma)^{1}}-4 v_{(\alpha \sigma)^{1}}$ & 0 & $2 v_{(\alpha \sigma)^{1}}$ & 0 \\
\hline $1 s \in\left(i^{-}\right)$ & $-4 v_{(\alpha \sigma)^{1}}$ & $v_{(\alpha \sigma)^{1}}$ & 0 & $2 v_{(\alpha \sigma)^{1}}$ \\
\hline \multirow[t]{2}{*}{$2 s \in\left(i^{-}\right)$} & $v_{(\alpha \sigma)^{1}}-4 v_{(\alpha \sigma)^{1}}$ & 0 & $v_{(\alpha \sigma)^{1}}$ & $v_{(\alpha \sigma)^{1}}$ \\
\hline & $\begin{array}{c}\gamma_{(i j)^{1}} \sum_{p} \gamma_{(i s)^{1}} \\
\left(1-\gamma_{(i p)^{1}}\right)\left(1-\gamma_{(j p)^{1}}\right) \\
\gamma_{(j s)^{1}} \gamma_{(s p)^{1}} v_{s p}^{\alpha \sigma}\end{array}$ & $\begin{array}{l}\gamma_{(i j)^{1}} \sum_{p} \gamma_{(i s)^{1}} \\
\left(1-\gamma_{(i p)^{1}}\right) \gamma_{(j p)^{1}} \\
\gamma_{(j s)^{1}} \gamma_{(s p)^{1}} v_{s p}^{\alpha \sigma}\end{array}$ & $\begin{array}{c}\gamma_{(i j)^{1}} \sum_{p} \gamma_{(i s)^{1}} \\
\gamma_{(i p)^{1}}\left(1-\gamma_{(j p)^{1}}\right) \\
\gamma_{(j s)^{1}} \gamma_{(s p)^{1}} v_{s p}^{\alpha \sigma}\end{array}$ & $\begin{array}{c}\gamma_{(i j)^{1}} \sum_{p} \gamma_{(i s)^{1}} \\
\gamma_{(i p)^{1}} \gamma_{(j p)^{1}} \\
\gamma_{(j s)^{1}} \gamma_{(s p)^{1}} v_{s p}^{\alpha \sigma}\end{array}$ \\
\hline $2 s \in\left(i^{+}\right)$ & $2 v_{(\alpha \sigma)^{1}}-v_{(\alpha \sigma)^{1}}$ & $2 v_{(\alpha \sigma)^{1}}$ & $-v_{(\alpha \sigma)^{1}}$ & $-v_{(\alpha \sigma)^{1}}$ \\
\hline $2 s \in(i)$ & $v_{(\alpha \sigma)^{1}}-2 v_{(\alpha \sigma)^{1}}$ & $v_{(\alpha \sigma)^{1}}$ & $-2 v_{(\alpha \sigma)^{1}}$ & $v_{(\alpha \sigma)^{1}}$ \\
\hline
\end{tabular}

TABLE II: Value of the intermediate sums to be calculated in equations (25) and (26) which depend on four point averages and nearest neighbour effective interactions, in the particular case of a fcc crystal. In each cell, the number in front of the positive nn effective interaction corresponds to the number of equivalent four-point averages weighted by the sign of the associated nn effective interaction when summed over $\mathrm{p}$ at fixed s. 
Figure 1 Dependence of $b$ with the ratio $\omega_{2} / \omega_{1}$ with $\omega_{4} / \omega_{0}=1$ and $\omega_{3} / \omega_{1}=0.5$ deduced from the first parameter set defined in section (IV). Line and full circles correspond to equilibrium Monte Carlo simulations, stars to the SCMF predictions, triangles to the five-frequency model of I.K. (Ishioka and Koiwa 1984), polygons to the five-frequency model of H.M. (Howard and Manning 1967) and crosses to the five-frequency model of L. (Lidiard 1960).

Figure 2 Dependence of $b$ with the ratio $\omega_{2} / \omega_{1}$ with $\omega_{4} / \omega_{0}=0.1$ and $\omega_{3} / \omega_{1}=1$. Line and full circles correspond to equilibrium Monte Carlo simulations (Belova and Murch 2003a), stars to the SCMF predictions, triangles to the five-frequency model of I.K. (Ishioka and Koiwa 1984), polygons to the five-frequency model of H.M. (Howard and Manning 1967) and crosses to the five-frequency model of L. (Lidiard 1960).

Figure 3 Dependence of $b$ with the ratio $\omega_{3} / \omega_{1}$ with $\omega_{4} / \omega_{0}=0.1$ and $\omega_{2} / \omega_{1}=0.1$ deduced from the second parameter set defined in section (IV). Line and full circles correspond to equilibrium Monte Carlo simulations, stars to the SCMF predictions, triangles to the fivefrequency model of I.K. (Ishioka and Koiwa 1984), polygons to the five-frequency model of H.M. (Howard and Manning 1967) and crosses to the five-frequency model of L. (Lidiard 1960).

Figure 4 Schematic diagram of a fcc crystal to illustrate the possible paths of a vacancy (empty circle) near a solute atom (black circle) after an exchange with a tracer atom A (grey circle). Cases a) and b) represent respectively the possible jumps offered to a vacancy after a first jump of type $\omega_{1}$ (resp. $\left.\omega_{0}\right)$. The thick arrow represents the most probable 
jump when the set of jump frequencies is the second set of section (IV) illustrated in figure (3) in the case of low values of $\omega_{3} / \omega_{1}$.

Figure 5 Dependence of $b$ with the ratio $\omega_{4} / \omega_{0}$ with $\omega_{3} / \omega_{1}=1$ and $\omega_{2} / \omega_{1}=1$ deduced from the third parameter set defined in section (IV). Line and full circles correspond to equilibrium Monte Carlo simulations, stars to the SCMF predictions, triangles to the five-frequency model of I.K. (Ishioka and Koiwa 1984), polygons to the five-frequency model of H.M. (Howard and Manning 1967) and crosses to the five-frequency model of L. (Lidiard 1960).

Figure 6 Schematic diagram of a fcc crystal to illustrate possible paths of a vacancy (empty circle) near a solute atom (black circle) after an exchange with a tracer atom A (grey circle). Cases a) and b) represent respectively the possible jumps offered to a vacancy after a first jump of type $\omega_{3}$ (resp. $\left.\omega_{0}\right)$. The thick arrow represents the most probable jump when the set of jump frequencies is the third one of section (IV) illustrated in figure (5) in the region of large values of $\omega_{4} / \omega_{0}$. 


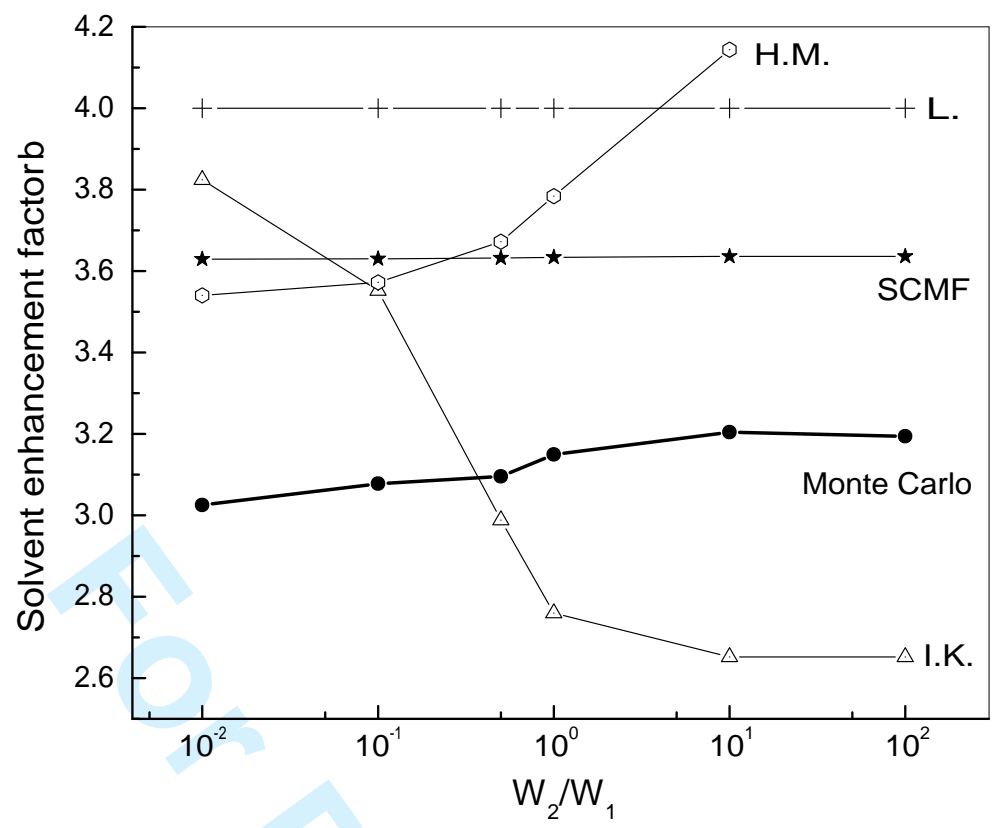

FIG. 1: 


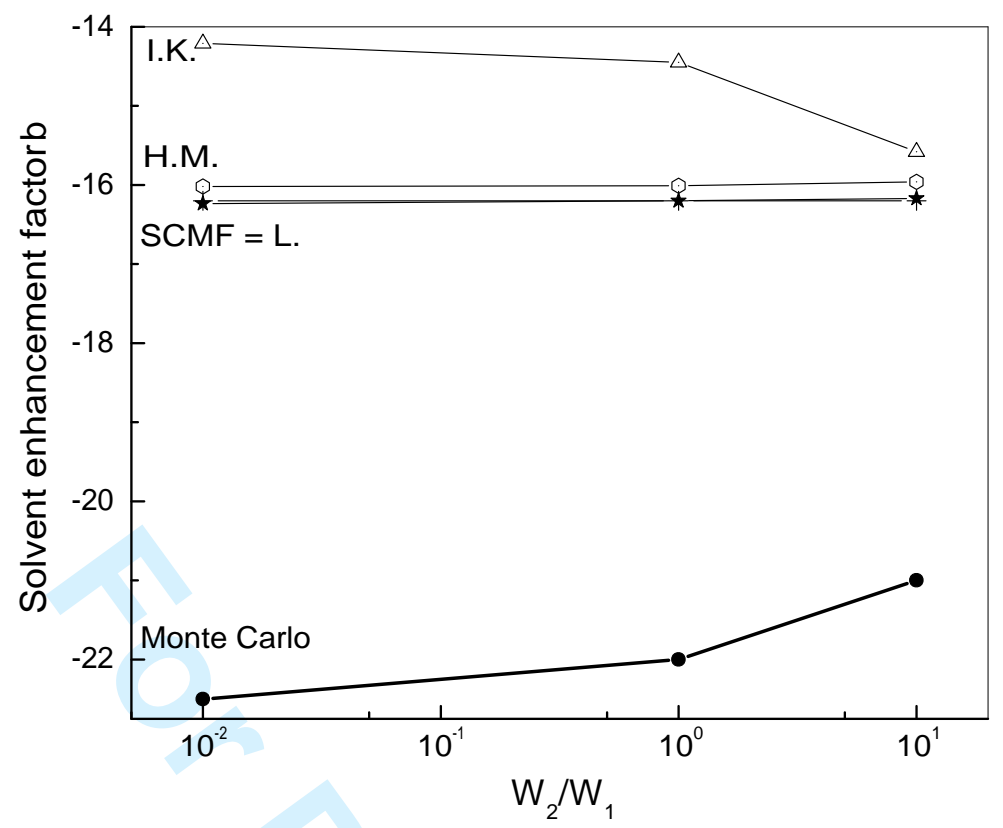

FIG. 2: 


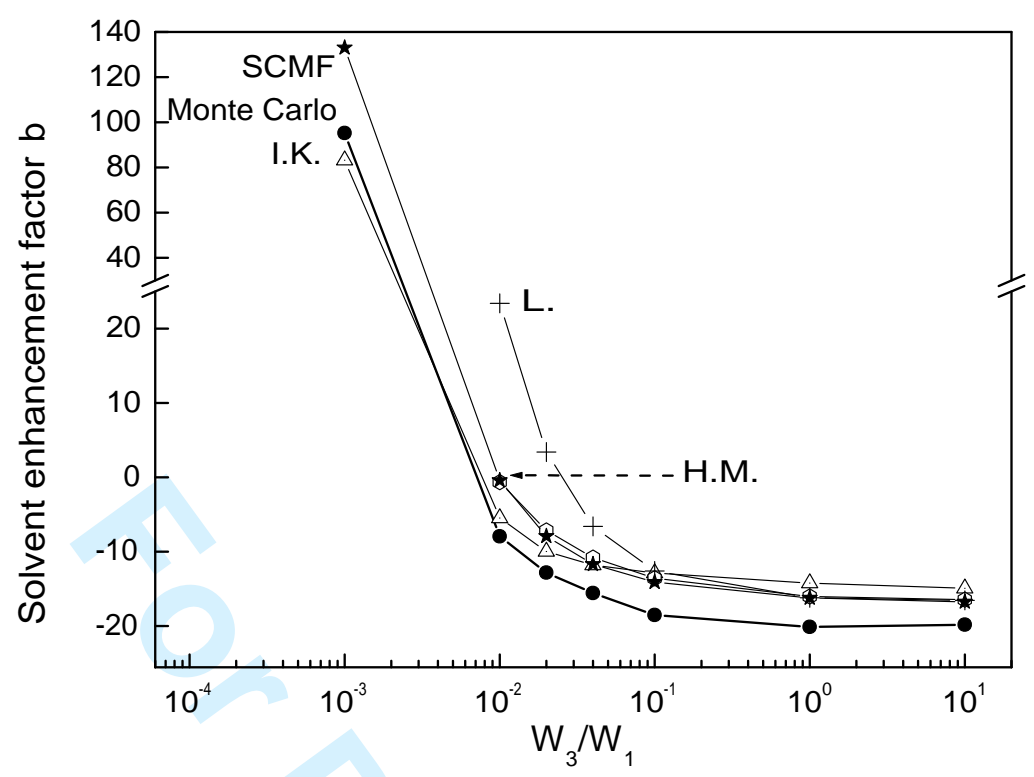

FIG. 3: 
a)
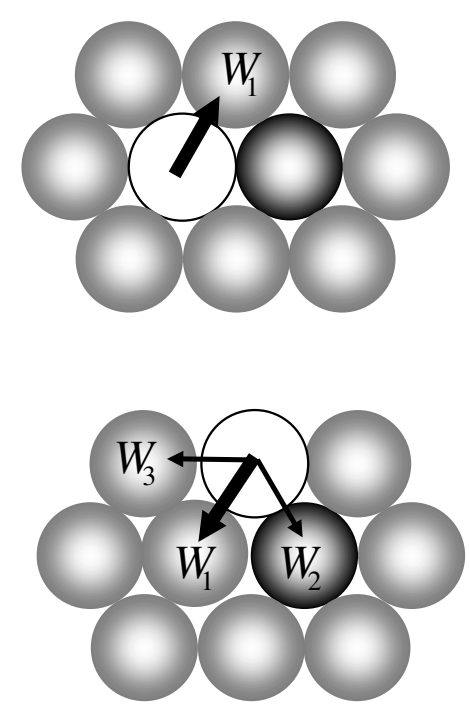

b)
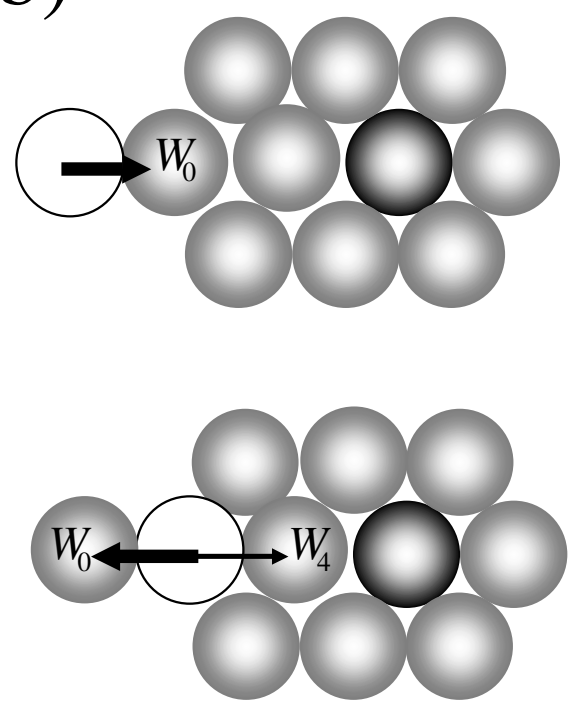

FIG. 4: 


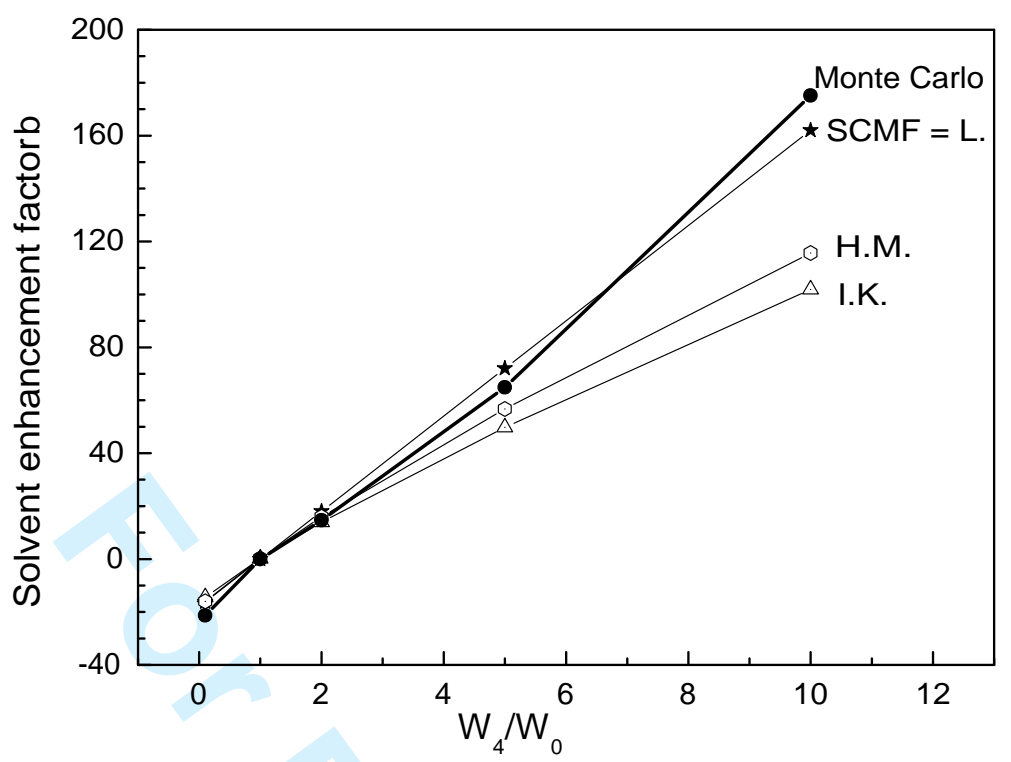

FIG. 5: 
a)
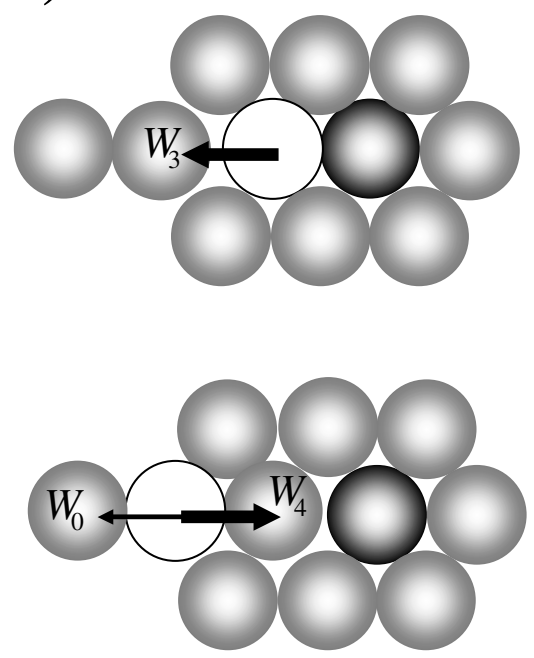

b)
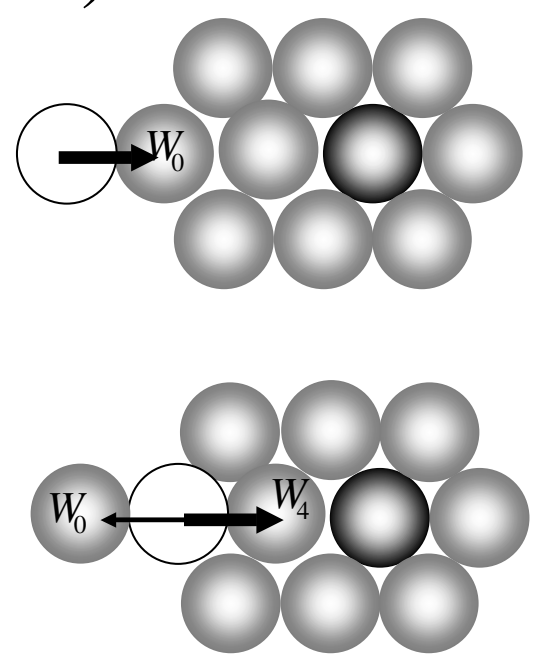

FIG. 6: 